\title{
Hydrologic Data for Urban Studies in the Austin, Texas Metropolitan Area, 1972
}

By E. E. WEHMEYER

UNITED STATES DEPARTMENT OF THE INTERIOR GEOLOGICAL SURVEY

Open-file report

Prepared in cooperation with the Texas Water Development Board June 1974 


\section{CONTENTS}

Int roduction-1..- 4

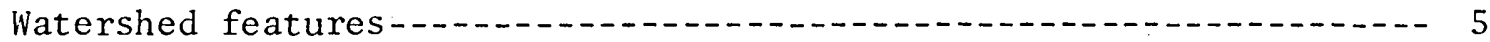

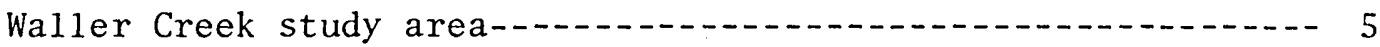

Wilbarger Creek study area-1... 7

Hydrologic instruments-_... 7

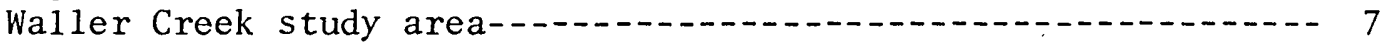

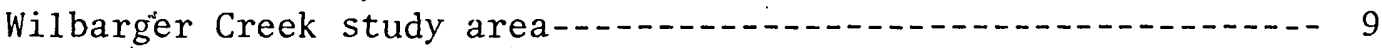

Summary of data for Waller Creek, 1972 water year-.............. 9

Summary of data for Wilbarger Creek, 1972 water year-_.........- 11

Compilation of data-1

Waller Creek at 38th Street, Austin, Tex.-.... 15

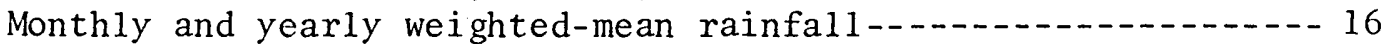

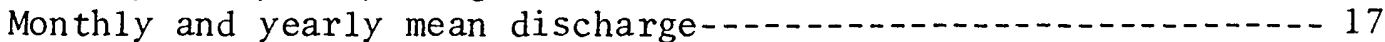

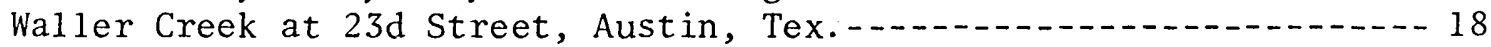

Monthly and yearly weighted-mean rainfall-_............... 19

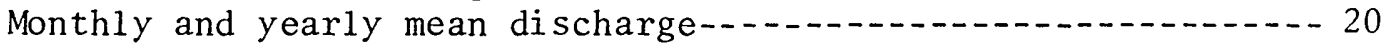

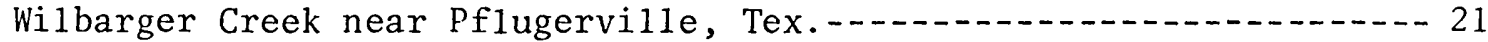

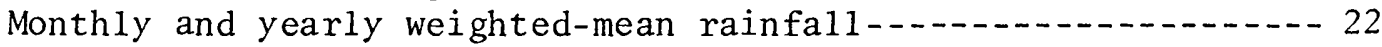

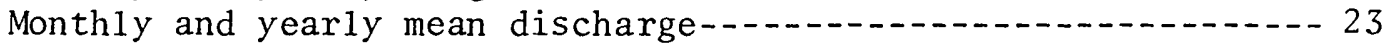

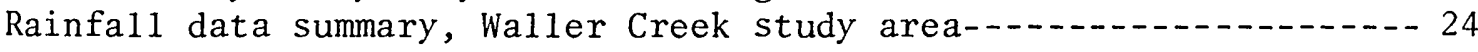

Rainfall data summary, Wilbarger Creek study area-............... 29

Storm rainfall and runoff records for:

Waller Creek at 38th Street

Storm of November 17-18, 1971-_...... 30

Hydrograph and mass curves-....... 32

Storm of May 1-2, 1972

Hydrograph and mass curves-...... 35

Waller Creek at $23 \mathrm{~d}$ Street

Storm of November 17-18, 1971_...... 36

Hydrograph and mass curves-_....... 38

Storm of May 1-2, 1972_-_._. 39

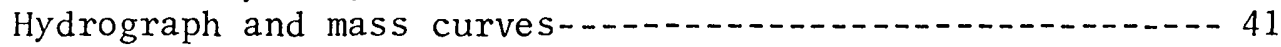

Wilbarger Creek near Pflugerville, Tex.

Storm of November 17-18, 1971-_. 42

Hydrograph and mass curves....... 44

Storm of December 5, 1971-_... 45

Hydrograph and mass curves-...... 46

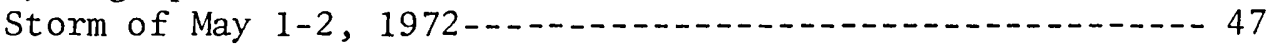

Hydrograph and mass curves.............. 49 


\section{ILLUSTRATIONS}

Page

Figure 1. Map showing the location of Waller Creek and

Wilbarger Creek study areas-........................ 6

2. Map showing the locations of hydrologic-instrument

installations in the Waller Creek study area-.....-- 8

3. Map showing the locations of hydrologic-instrument

installations in the Wilbarger Creek study area-...-.- 10

TABLE

Table 1. Storm rainfall-runoff data, 1972 water year-_......... 12 
HYDROLOGIC DATA FOR URBAN STUDIES IN THE

AUSTIN, TEXAS METROPOLITAN AREA

1972

By

E. E. Wehmeyer

U.S. Geological Survey

\section{INTRODUCTION}

The U.S. Geological Survey, in cooperation with the Texas Water Development Board, began hydrologic studies in the Austin urban area in 1954. The objectives of this project are as follows:

1. To determine the effects of progressive urbanization on infiltration, rates of peak discharge, and rainfallrunoff relations in the Waller Creek watershed.

2. To provide rainfall and runoff data from the rural Wilbarger Creek watershed to be used for comparative purposes in determining the effects of existing and progressive urbanization in the Waller Creek watershed.

3. To provide applied research facilities for studies at The University of Texas at Austin.

The purpose of this report is to present rainfall and runoff data for the Waller Creek and Wilbarger Creek study areas for the 1972 water year (October 1, 1971 to September 30, 1972).

To facilitate the publication and distribution of this report at the earliest feasible time, certain material has been included that does not conform to the formal publication standards of the U.S. Geological Survey. 
For those readers interested in using the metric system, metric equivalents of English units of measurements are given in parentheses. The English units used in this report may be converted to metric units by the following conversion factors:

\begin{tabular}{lcclc}
\hline Unit & $\begin{array}{c}\text { Abbrevi- } \\
\text { ation }\end{array}$ & Multiply by & $\begin{array}{c}\text { To obtain } \\
\text { Unit }\end{array}$ & $\begin{array}{c}\text { Abbrevi- } \\
\text { ation }\end{array}$ \\
\hline inch & in & 25.4 & millimeter & $\mathrm{mm}$ \\
foot & $\mathrm{ft}$ & 0.3048 & meter & $\mathrm{m}$ \\
mile & $\mathrm{mi}$ & 1.609 & kilometer & $\mathrm{km}$ \\
square mile & $\mathrm{mi}^{2}$ & 2.590 & square kilometer & $\mathrm{km}^{2}$ \\
cubic foot & $\mathrm{ft}^{3} / \mathrm{s}$ & 28.32 & cubic decimeter per second & $\mathrm{dm} / \mathrm{s}$ \\
per second & & 0.02832 & cubic meter per second & $\mathrm{m} / \mathrm{s}$ \\
foot per mile & $\mathrm{ft} / \mathrm{mi}$ & $0.3048 / 1.609$ & meter per kilometer & $\mathrm{m} / \mathrm{km}^{3}$ \\
acre-foot & ac- $\mathrm{ft}$ & 1233 & cubic meter & $\mathrm{m}$ \\
& & $1.233 \times 10^{-3}$ & cubic hectometer & $\mathrm{hm}$ \\
\hline
\end{tabular}

\section{WATERSHED FEATURES}

Waller Creek Study Area

The Waller Creek drainage area (fig. 1) lies entirely within the city of Austin, with the headwaters originating in the northern part of the city. The creek flows south for 6.6 miles (10.6 kilometers) to the Colorado River. Storm sewers and street gutters divert runoff both into and out of the natural drainage area.

Throughout the year, low flow is partially sustained by return flow of city water from industrial and residential users. Low flow during the summer months is partly sustained by drainage from municipal and private swimming pools. 


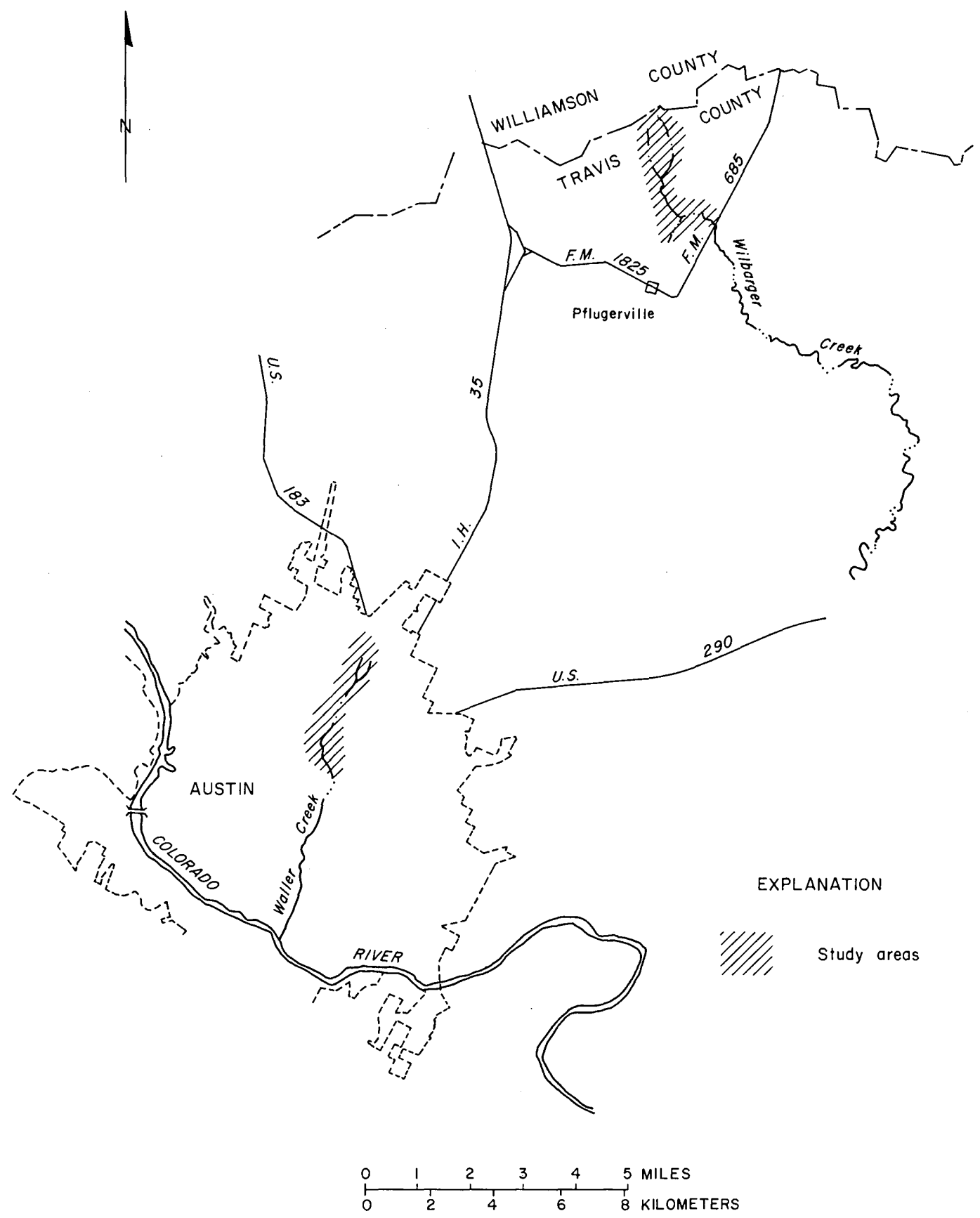


In $1954,20.6$ percent of the drainage area above $23 \mathrm{~d}$ Street was impervious cover, i.e., streets, sidewalks, roofs, etc; this cover had increased to 28.6 percent by 1962 and to 35.2 percent by 1966 . Impervious cover in the drainage area above 38 th Street increased from 13 percent in 1954 to 21 percent in 1962 and to 33.3 percent in 1966 . Soil in the watershed is predominantly clay, which is underlain by Cretaceous chalk that crops out along most of the channel.

The slope index for waller Creek is 48.6 feet per mile (9.2 meters per kilometer) at 38 th Street and 47.3 feet per mile $(9.0$ meters per kilometer) at $23 \mathrm{~d}$ Street. These indices were computed by determining the difference in elevation between the two points that were 85 and 10 percent of the main channel distance upstream from the gaging station, and then dividing by the distance between the two points.

Records of the Environmental Data Service show that the mean-annual rainfall (based on the period 1931-60), approximately 1.5 miles (2 kilometers) east of the watershed, is 32.58 inches ( 827.5 millimeters). Rainfall is generally well distributed throughout the year; however, individual storms may cause serious flooding in any season. The major storms usually occur during the months of April-May and September-October.

\section{Wilbarger Creek Study Area}

The headwaters of Wilbarger Creek originate in Travis County near the Williamson County line (fig. 1). The creek flows southeasterly about 40 miles (64 kilometers) to the Colorado River. The Wilbarger Creek study area is about 15 miles (24 kilometers) north of the city of Austin:

The Soil in the watershed is predominantly clay, which is underlain by Cretaceous chalk that crops out at many places along the channel. The principal land use is farming and ranching.

The slope index, calculated by the 85-10 percent method, is 39.2 feet per mile ( 7.4 meters per kilometer). Runoff characteristics are slightly affected by farming practices, stock tanks, and vegetation.

\section{HYDROLOGIC INSTRUMENTS}

\section{Waller Creek Study Area}

Instruments to collect rainfall and runoff data in the Waller Creek study area consist of a network of three recording and three nonrecording rain gages and two stream-gaging stations. Figure 2 shows the locations of instruments in the study area. 


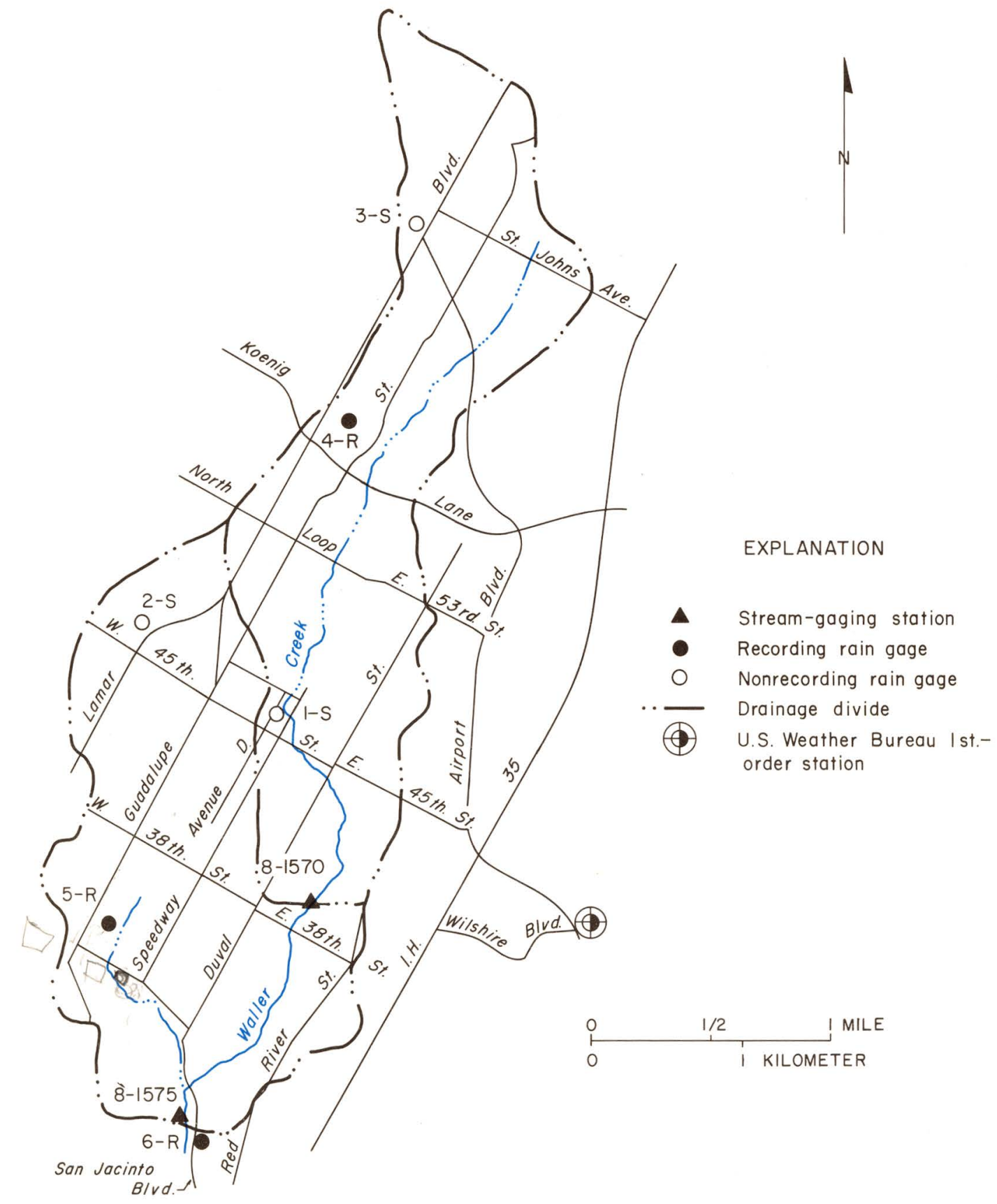

Base from General Highway Map of Texas

FIGURE 2.-- Locations of hydrologic-instrument installations in the Woller Creek study area 
The rain gages are distributed in the study area to measure the total rainfall and to define rainfall intensities on the watershed.

One stream-gaging station is maintained at 38th Street and the other at $23 \mathrm{~d}$ Street. Low-water concrete controls were constructed at each stream-gaging station to stabilize the stage-discharge relationship. Records at the 38th Street station began Apri1 5, 1955, and at the 23d Street station on December 23, 1954. Pertinent station information and records of daily, monthly, and yearly runoff for the 1972 water year for both stations are included in the section "Compilation of Data".

\section{Wilbarger Creek Study Area}

Instruments to collect rainfall and runoff data in the Wilbarger Creek study area consist of a network of three recording rain gages and one stream-gaging station. Figure 3 shows the locations of instruments in the study area.

The rain gages are distributed in the study area to measure the total rainfall and to define rainfall intensities on the watershed.

A continuous-recording stream-gaging station equipped with a concrete low-water control is maintained on Wilbarger Creek. Records at this station began August 9, 1963. Pertinent station information and records of daily, monthly, and yearly runoff for the 1972 water year are included in the section "Compilation of Data".

\section{SUMMARY OF DATA FOR WALLER CREEK, 1972 WATER YEAR}

The weighted-mean rainfall in the study area upstream from 38 th Street was 35.41 inches (899.4 millimeters), 9 percent above the mean annual rainfall for Austin of 32.58 inches (827.5 millimeters). Mean daily discharge was $1.60 \mathrm{ft} / \mathrm{s}$ (cubic feet per second) or $45.3 \mathrm{dm} / \mathrm{s}$ (cubic decimeters per second); annual runoff was 9.40 inches (238.8 millimeters), or 27 percent of rainfall.

The weighted-mean rainfall in the study area upstream from $23 \mathrm{~d}$ Street was 34.94 inches ( 887.5 millimeters), 7 percent above the mean annual rainfall for Austin. Mean daily discharge was $3.44 \mathrm{ft}^{3} / \mathrm{s}$ $(94.4 \mathrm{dm} / \mathrm{s})$; annual runoff was 11.34 inches (288.0 millimeters), or 32 percent of rainfall. 


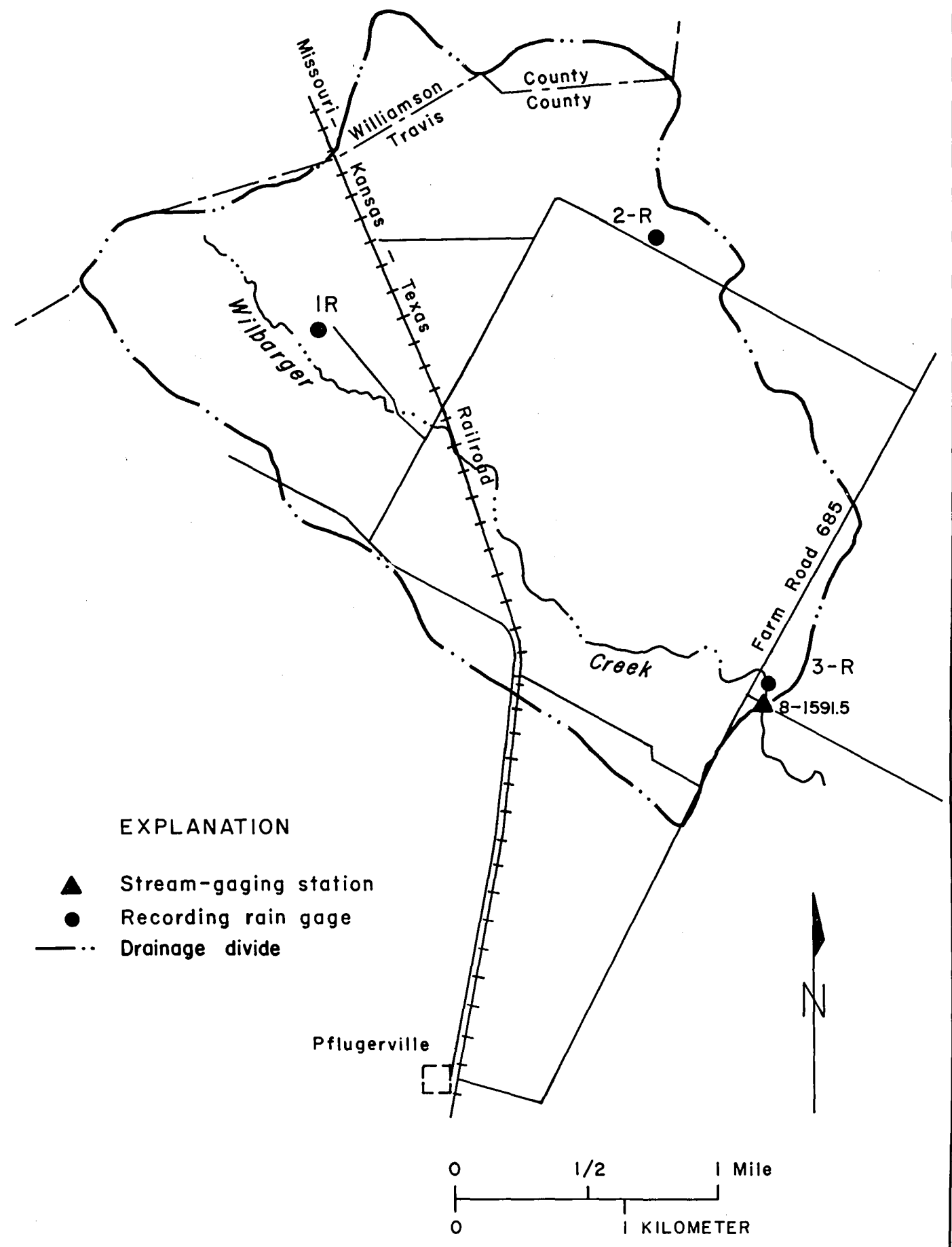

Base from General Highway Map of Texas

FIGURE 3.-- Locations of hydrologic-instrument installations in the Wilbarger Creek study area 
A storm event is defined as a period of rainfall separated by at least 6 hours from other rainfall. Storms are selected for detailed rainfall-runoff computations on the basis of rainfall totals and distribution, the peak discharge produced from the rainfall, and the assurance of good rainfall and runoff records for the storm periods selected.

Two storm periods, November 17-18, 1971 and May 1-2 1972, were selected for analysis. A summary of rainfall-runoff data for each storm is shown in table 1. Computations with hydrograph and mass curves for each storm are included in the section "Compilation of Data".

SUMMARY OF DATA FOR WILBARGER CREEK, 1,972 WATER YEAR

Weighted-mean rainfall in the study area was 30.35 inches (770.9 millimeters), 7 percent below the mean annual rainfall for Austin. Mean daily discharge was $0.96 \mathrm{ft}^{3} / \mathrm{s}\left(27.2 \mathrm{dm}^{3} / \mathrm{s}\right)$; annual runoff was 2.83 inches (71.9 millimeters), or 9 percent of rainfall.

Three storm periods, November 17-18, 1971, December 5, 1971, and May 1-2, 1972 were selected for analysis. A summary of rainfall-runoff data for each storm is shown in table 1. Computations with hydrograph and mass curves for each storm are included in the section "Compilation of Data". 
ANNUAL STORM RAINFALL-RUNOFF SUMMARY DATA

Table 1.--Storm rainfall-runoff data, 1972 water year

\begin{tabular}{|c|c|c|c|c|c|c|c|c|}
\hline \multirow[b]{2}{*}{ Date of Storm } & \multicolumn{5}{|c|}{ Rainfall (inches) } & \multirow{2}{*}{$\begin{array}{l}\text { Runoff } \\
\text { (inches) }\end{array}$} & \multirow{2}{*}{$\begin{array}{l}\text { Ratio } \\
\text { runoff to } \\
\text { rainfall }\end{array}$} & \multirow{2}{*}{$\begin{array}{l}\text { Maximum } \\
\text { discharge } \\
\left(\mathrm{ft}^{3} / \mathrm{s}\right)\end{array}$} \\
\hline & $\begin{array}{l}\text { Duration } \\
\text { (hours) }\end{array}$ & Total & \multicolumn{3}{|c|}{ Maximum increment } & & & \\
\hline \multicolumn{9}{|c|}{$\begin{array}{c}\text { Waller Creek at } 38 \text { th St., Austin, Texas } \\
\text { (Drainage area, } 2.31 \mathrm{mi}^{2} \text { ) }\end{array}$} \\
\hline Nov. $17-18,1971$ & 15.5 & 3.86 & .55 & 1.00 & 1.08 & .91 & .24 & 388 \\
\hline May $1-2,1972$ & 12.2 & 2.79 & .79 & 1.49 & 2.01 & 1.10 & .39 & 1,400 \\
\hline & & & & & & & & \\
\hline & & & & & & & & \\
\hline & & & & & & & & \\
\hline \multicolumn{9}{|c|}{$\begin{array}{c}\text { Waller Creek at } 23 r d \text { St., Austin, Texas } \\
\text { (Drainage area } 4.13 \mathrm{mi}^{2} \text { ) }\end{array}$} \\
\hline Nov. $17-18,1971$ & 16.2 & 3.68 & .52 & .82 & 1.10 & 1.00 & .27 & 765 \\
\hline May $1-2, \quad 1972$ & 12.5 & 2.81 & .78 & 1.36 & 1.98 & 1.06 & .38 & 2,160 \\
\hline & & & & & & & & \\
\hline & & & & & & & & \\
\hline & & & & & & & & \\
\hline & & & & & & & & \\
\hline
\end{tabular}


ANNUAL STORM RAINFALL-RUNOFF SUMMARY DATA

Table 1.--Storm rainfall-runoff data, 1972 water year

\begin{tabular}{|c|c|c|c|c|c|c|c|c|}
\hline \multirow{3}{*}{ Date of Storm } & \multicolumn{5}{|c|}{ Rainfall (inches) } & \multirow{3}{*}{$\begin{array}{l}\text { Runoff } \\
\text { (inches) }\end{array}$} & \multirow{3}{*}{$\begin{array}{l}\text { Ratio } \\
\text { runoff to } \\
\text { rainfall }\end{array}$} & \multirow{3}{*}{$\begin{array}{l}\text { Maximum } \\
\text { discharge } \\
\left(\mathrm{ft}^{3} / \mathrm{s}\right)\end{array}$} \\
\hline & \multirow{2}{*}{$\begin{array}{l}\text { Duration } \\
\text { (hours) }\end{array}$} & \multirow{2}{*}{ Total } & \multicolumn{3}{|c|}{ Maximum increment } & & & \\
\hline & & & 15-minute & 30-minute & 60-minute & & & \\
\hline \multicolumn{9}{|c|}{$\begin{array}{l}\text { Wilbarger Creek near Pflugerville, Texas } \\
\text { (Drainage area, } 4.61 \mathrm{mi}^{2} \text { ) }\end{array}$} \\
\hline Nov. $17-18,197]$ & 16.5 & 4.22 & .69 & 1.13 & 1.43 & .41 & .10 & 563 \\
\hline Dec. 5, 1971 & 10.5 & 1.12 & .18 & .36 & .43 & .25 & .22 & 158 \\
\hline May $1-2,1972$ & 5.8 & 2.70 & .45 & .79 & 1.27 & .32 & .12 & 464 \\
\hline & & & & & & & & \\
\hline & & & & & & & & \\
\hline & & & & & & & & \\
\hline
\end{tabular}

\begin{tabular}{l|l|l|l|l|l|l|l|l}
\hline & & & & & & & & \\
\hline & & & & & & & & \\
\hline & & & & & & & & \\
\hline & & & & & & & & \\
\hline & & & & & & & & \\
\hline
\end{tabular}




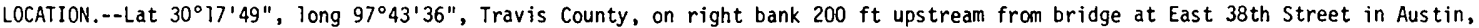
1.1 miles upstream from West Branch of Waller Creek, and 3.3 miles upstream from Colorado River.

DRAINAGE AREA.--2.31 sq mi.

PERIOD OF RECORD.--April 1955 to current year.

GAGE.--Water-stage recorder and concrete control. Datum of gage is $555.44 \mathrm{ft}$ above mean sea level.

AVERAGE DISCHARGE.--17 years, $1.66 \mathrm{cfs}$ (9.76 inches per year, 1,200 acre-ft per year).

EXTREMES.--Current year: Maximum discharge, 1,400 cfs May 2 (gage height, $7.09 \mathrm{ft}$ ); minimum daily, $0.08 \mathrm{cfs}$ Sept. $16,18,20$.

Period of record: Maximum discharge, 1,970 cfs 0ct. 29, 1960 (gage height, $7.77 \mathrm{ft}$ ); no flow for many days in $1955-57,1964$.

REMARKS.--Records good. Flow slightly regulated at times by a small reservoir at Holy Cross High School (formerly St. Mary's Academy) on East $41 \mathrm{st}$ Street and a small swimming pool at the school which is drained into the creek every week or two during the summer. Water from other swimming pools also drain into the creek. Station is part of hydrologic research project to study rainfallrunoff relation for smali urban areas. Two recording and three nonrecording rain gages are distributed in the area so that rainfall on the watershed can be determined:

DISCHARGE, IN CUBIC FEET PER SECOND, WATEF YEAR OCTOBER 1971 TO SEPTEMBER 1972

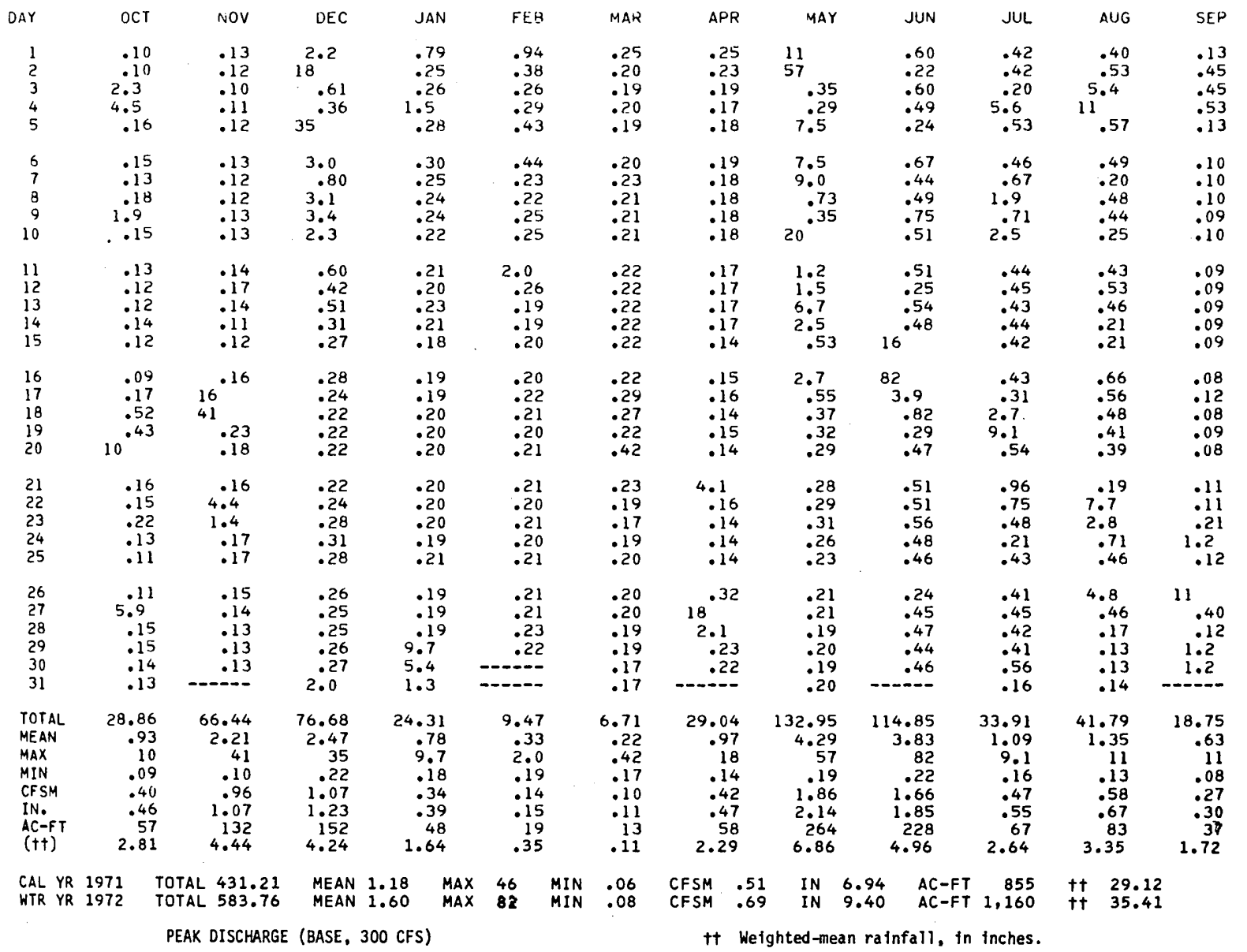

DATE TIME G.HT. DISCHARGE DATE TIME G.HT. DISCHARGE

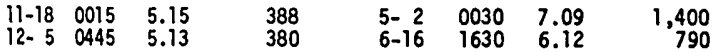

1/This table reproduced from U.S. Geological Survey, 1972, Water Resources Data for Texas, Part 1, Surface Water Records. 
[Drainage area, 2.3. square miles]

\begin{tabular}{|c|c|c|c|c|c|c|c|c|c|c|c|c|c|c|}
\hline \multicolumn{12}{|c|}{5} & \multirow{2}{*}{$\begin{array}{l}10-26489-5 \\
\text { SEPT. }\end{array}$} & \multicolumn{2}{|c|}{ U. S. COVERAMERT PRATTING OFICE } \\
\hline YEAR & ост. & NOV. & $\overline{\mathrm{DEC} .}$ & JAN. & FEB. & MAR. & APR. & MAY & JUNE & JULY & AUG. & & ANNUAL & Cal. Yr. \\
\hline 1956 & - & - & - & - & - & 0.49 & 0.60 & 3.00 & 1.40 & 0.44 & 1.37 & 0.21 & - & \\
\hline 1957 & 1.31 & 2.04 & 2.08 & 0.49 & 2.73 & 3.49 & 9.37 & 7.07 & 5.06 & .57 & .03 & 6.85 & 41.09 & 50.37 \\
\hline 1958 & 10.21 & 3.24 & 1.26 & 3.06 & 6.81 & 2.65 & 5.02 & 3.34 & 4.62 & 2.15 & .84 & 7.20 & 50.40 & 43.99 \\
\hline 1959 & 6.18 & .92 & 1.20 & .44 & 2.80 & .26 & 4.86 & 2.20 & 3.30 & 4.39 & 5.07 & 4.00 & 35.62 & 38.60 \\
\hline 1960 & 7.41 & 1.68 & 2.19 & 1.22 & 2.43 & 1.30 & 1.12 & 1.03 & 4.25 & 2.52 & 3.52 & 1.46 & 30.13 & 37.50 \\
\hline 1961 & 12.11 & 2.13 & 4.41 & 1.48 & 5.03 & .72 & .15 & 1.21 & 11.95 & 8.37 & .76 & 3.89 & 52.21 & 38.67 \\
\hline 1962 & 1.01 & 2.36 & 1.74 & .69 & .99 & 1.19 & 4.87 & 1.43 & 8.71 & .03 & 3.38 & 4.19 & 30.59 & 33.82 \\
\hline 1963 & 3.96 & 1.26 & 3.12 & .53 & 2.64 & .24 & 2.77 & 1.42 & 3.59 & .10 & .47 & 1.30 & 21.40 & 16.92 \\
\hline 1964 & .91 & 1.68 & 1.27 & 2.85 & 1.51 & 2.25 & 1.47 & 1.91 & 5.71 & 1.19 & 1.62 & 7.91 & 30.28 & 34.36 \\
\hline 1965 & 4.10 & 2.79 & 1.05 & 4.29 & 5.80 & 1.34 & 1.72 & 9.22 & 1.85 & .32 & .69 & 6.43 & 39.60 & 43.64 \\
\hline 1966 & 3.50 & 3.33 & 5.15 & 1.42 & 2.73 & .59 & 4.29 & 3.49 & .94 & .35 & 7.04 & 3.70 & 36.53 & 26.25 \\
\hline 1967 & .96 & .08 & .66 & .15 & 1.43 & .91 & 4.27 & 3.77 & 0 & .99 & 5.03 & 6.71 & 24.96 & 36.13 \\
\hline 1968 & 4.80 & 4.55 & 3.52 & 8.37 & 1.63 & 2.77 & 2.49 & 6.68 & 2.90 & 2.90 & .77 & 2.98 & 44.36 & 38.35 \\
\hline 1969 & .58 & 5.59 & .69 & .40 & 4.16 & 2.90 & 4.92 & 2.91 & 2.13 & .30 & 4.52 & 1.15 & 30.25 & 32.37 \\
\hline 1970 & 3.26 & .88 & 4.84 & 1.98 & 6.03 & 2.93 & 1.26 & 7.58 & .21 & .31 & 1.56 & 4.98 & 35.82 & 31.87 \\
\hline 1971 & 4.92 & 0 & .11 & .04 & .98 & .59 & 1.08 & 1.47 & 2.16 & 1.56 & 7.09 & 2.66 & 22.66 & 29.12 \\
\hline 1972 & 2.81 & 4.44 & 4.24 & 1.64 & .35 & .11 & 2.29 & 6.86 & 4.96 & 2.64 & 3.35 & 1.72 & 35.41 & \\
\hline & & & & & & & & & & & & & & \\
\hline & & & & & & & & & & & & . & & \\
\hline & & & & & & & & & & & & & & \\
\hline & & & & & & & & & & & & & & \\
\hline & & & & & & & & & & & & & & \\
\hline & & & & & & & & & & & & & & \\
\hline & & & & & & & & & & & & & & \\
\hline & & & & & & & & & & & & & & \\
\hline
\end{tabular}


Monthly and ${ }_{\wedge}$ yearly mean discharge, in

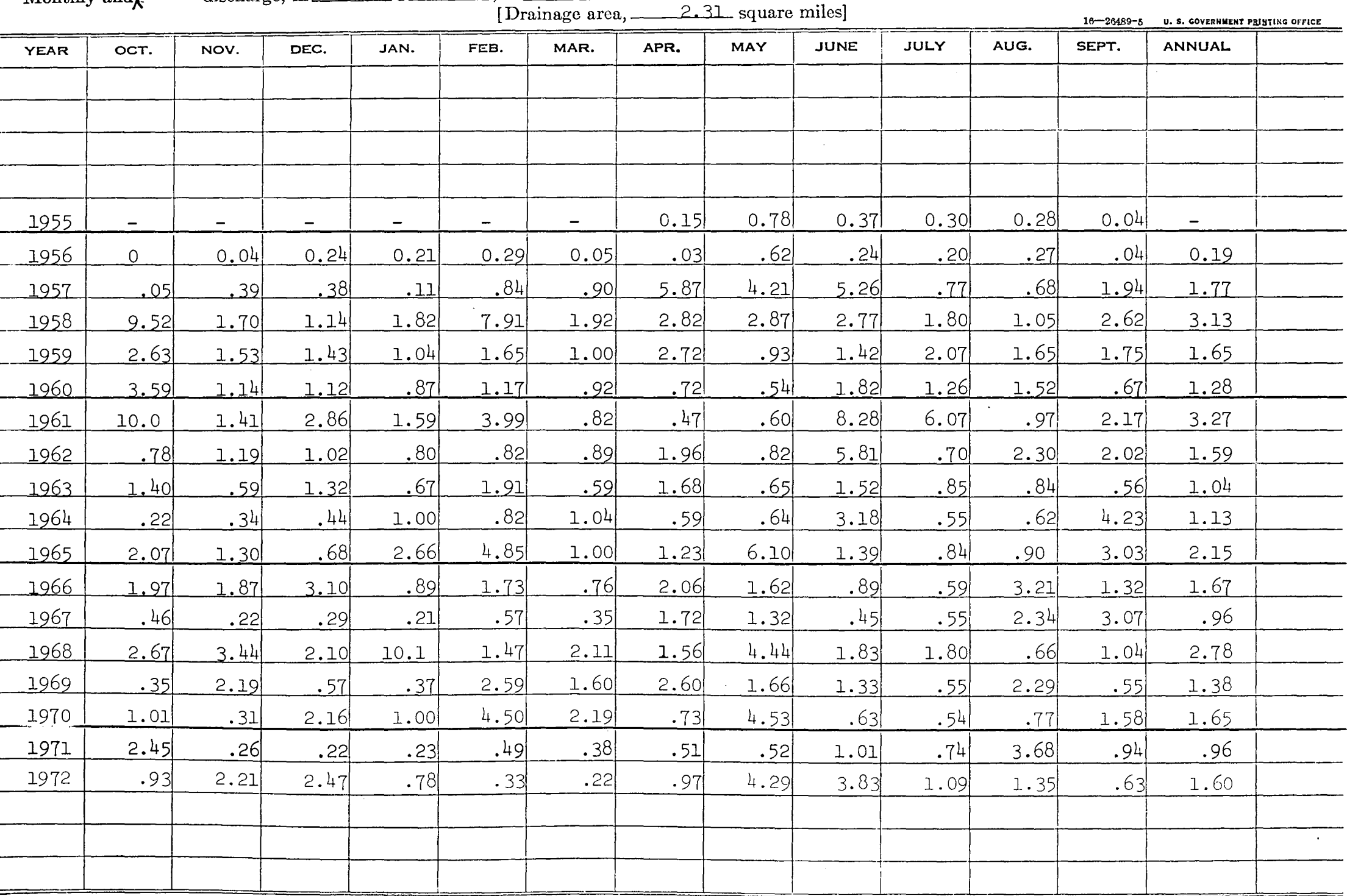


08157500 Waller Creek at 23d Street, Austin, Tex. 1/

LOCATION.--Lat $30^{\circ} 17^{\prime} 08^{\prime \prime}$, long $97^{\circ} 44^{\prime} 01^{\prime \prime}$, Travis County, on San Jacinto Boulevard, $50 \mathrm{ft}$ upstream from bridge on East $23 \mathrm{~d}$ Street in Austin, and 2.1 miles upstream from Colorado River.

DRAINAGE AREA. $--4.13 \mathrm{sq} \mathrm{mi}$.

PERIOD OF RECORD.--December 1954 to current year.

GAGE.--Water-stage recorder and concrete control. Datum of gage is $509.95 \mathrm{ft}$ above mean sea level.

AVERAGE DISCHARGE.--17 years, $3.58 \mathrm{cfs}$ ( 11.77 inches per year, 2,590 acre-ft per year).

EXTREMES ---Current year: Maximum discharge, 2,160 cfs May 2 (gage height, $6.91 \mathrm{ft}$ ); minimum daily, $0.42 \mathrm{cfs}$ Nov. 7.

Period of record: Maximum discharge, 3,710 cfs 0ct. 29, 1960 (gage height, $7.96 \mathrm{ft}$ ); minimum daily, $0.2 \mathrm{cfs}$ at times in 1955-57.

Maximum flood since 1885 occurred Apr. 22, 1915, stage unknown.

REMARKS.--Records good. Some regulation by small dam upstream. Diversion of city water into channel during the surmer months from municipal and private swimming pools. Some diversions into and out of drainage area by storm sewers. Station is part of a hydrologic research project to study rainfall-runoff relation for small urban areas. Three recording and three nonrecording rain gages located in watershed.

DISCHARGE, IN CUHIC FEET PER SECONO, WATER YEAR OCTOBER 1971 TO SEPTEMBER 1972

\begin{tabular}{|c|c|c|c|c|c|c|c|c|c|c|c|c|}
\hline DAY & OCT & NOV & DEC & $J A N$ & FEB & MAR & APR & MAY & JINN & JUL & AUG & SFP \\
\hline $\begin{array}{l}1 \\
2 \\
3 \\
4 \\
5\end{array}$ & $\begin{array}{c}.81 \\
.77 \\
4.5 \\
7.9 \\
.87\end{array}$ & $\begin{array}{l}.56 \\
.59 \\
.54 \\
.58 \\
.58\end{array}$ & $\begin{array}{c}4.3 \\
33 \\
1.3 \\
62\end{array}$ & $\begin{array}{c}1.6 \\
.69 \\
.90 \\
3.0 \\
.82\end{array}$ & $\begin{array}{c}2.2 \\
1.1 \\
.89 \\
.99 \\
1.3\end{array}$ & $\begin{array}{r}1.0 \\
.81 \\
.85 \\
.86 \\
.71\end{array}$ & $\begin{array}{l}.70 \\
.72 \\
.86 \\
.80 \\
.94\end{array}$ & $\begin{array}{c}17 \\
101 \\
1.4 \\
1.2 \\
14\end{array}$ & $\begin{array}{l}1.4 \\
1.1 \\
1.2 \\
1.2 \\
1.1\end{array}$ & $\begin{array}{r}1.2 \\
1.2 \\
1.2 \\
11 \\
1.4\end{array}$ & $\begin{array}{c}.95 \\
1.6 \\
9.6 \\
27 \\
1.3\end{array}$ & $\begin{array}{l}.86 \\
1.0 \\
1.1 \\
1.2 \\
.99\end{array}$ \\
\hline $\begin{array}{r}6 \\
7 \\
8 \\
9 \\
10\end{array}$ & $\begin{array}{c}.82 \\
.77 \\
1.2 \\
5.0 \\
.74\end{array}$ & $\begin{array}{l}.63 \\
.42 \\
.58 \\
.72 \\
.76\end{array}$ & $\begin{array}{l}5.5 \\
1.6 \\
6.1 \\
6.7 \\
3.8\end{array}$ & $\begin{array}{l}.88 \\
.87 \\
.84 \\
.96 \\
1.1\end{array}$ & $\begin{array}{l}1.3 \\
1.1 \\
1.1 \\
1.1 \\
1.3\end{array}$ & $\begin{array}{l}1.1 \\
1.2 \\
1.1 \\
1.0 \\
.96\end{array}$ & $\begin{array}{c}1.0 \\
1.0 \\
.91 \\
.92 \\
1.0\end{array}$ & $\begin{array}{l}14 \\
20 \\
2.1 \\
1.4 \\
37\end{array}$ & $\begin{array}{l}1.3 \\
1.3 \\
1.5 \\
2.5 \\
1.6\end{array}$ & $\begin{array}{l}1.3 \\
1.5 \\
2.4 \\
1.6 \\
3.3\end{array}$ & $\begin{array}{l}1.2 \\
1.1 \\
1.1 \\
1.2 \\
1.2\end{array}$ & $\begin{array}{l}.85 \\
.89 \\
.88 \\
.85 \\
.85\end{array}$ \\
\hline $\begin{array}{l}11 \\
12 \\
13 \\
14 \\
15\end{array}$ & $\begin{array}{l}.72 \\
.75 \\
.72 \\
.94 \\
.83\end{array}$ & $\begin{array}{l}.62 \\
.73 \\
.64 \\
.60 \\
.76\end{array}$ & $\begin{array}{l}1.3 \\
1.2 \\
1.4 \\
1.3 \\
1.2\end{array}$ & $\begin{array}{l}.90 \\
.93 \\
.92 \\
.77 \\
.68\end{array}$ & $\begin{array}{l}4.0 \\
1.2 \\
.93 \\
1.0 \\
1.0\end{array}$ & $\begin{array}{r}.97 \\
.90 \\
.95 \\
.99 \\
1.0\end{array}$ & $\begin{array}{c}1.0 \\
1.1 \\
.99 \\
.98 \\
.87\end{array}$ & $\begin{array}{r}3.3 \\
3.8 \\
17 \\
5.9 \\
1.9\end{array}$ & $\begin{array}{l}1.4 \\
1.3 \\
1.6 \\
1.4 \\
17\end{array}$ & $\begin{array}{l}1.1 \\
1.3 \\
1.2 \\
1.2 \\
1.1\end{array}$ & $\begin{array}{l}1.2 \\
1.3 \\
1.1 \\
1.1 \\
.95\end{array}$ & $\begin{array}{l}.93 \\
.93 \\
1.2 \\
.98 \\
1.0\end{array}$ \\
\hline $\begin{array}{l}16 \\
17 \\
18 \\
19 \\
20\end{array}$ & $\begin{array}{l}.74 \\
1.1 \\
1.4 \\
1.7 \\
21\end{array}$ & $\begin{array}{l}31^{.70} \\
80 \\
.88 \\
.76\end{array}$ & $\begin{array}{r}1.0 \\
.83 \\
.74 \\
.84 \\
.93\end{array}$ & $\begin{array}{l}.67 \\
.85 \\
.99 \\
1.1 \\
1.1\end{array}$ & $\begin{array}{c}1.0 \\
.98 \\
.96 \\
.85 \\
.89\end{array}$ & $\begin{array}{r}.92 \\
.99 \\
.96 \\
.81 \\
1.5\end{array}$ & $\begin{array}{l}.86 \\
.83 \\
.91 \\
.93 \\
.88\end{array}$ & $\begin{array}{l}6.3 \\
1.9 \\
1.5 \\
1.3 \\
1.2\end{array}$ & $\begin{array}{r}132 \\
6.6 \\
2.2 \\
1.5 \\
1.3\end{array}$ & $\begin{array}{r}1.0 \\
1.8 \\
3.3 \\
14 \\
1.4\end{array}$ & $\begin{array}{l}1.3 \\
1.2 \\
1.4 \\
2.5 \\
1.1\end{array}$ & $\begin{array}{r}.91 \\
.90 \\
.99 \\
.93 \\
.98\end{array}$ \\
\hline $\begin{array}{l}21 \\
22 \\
23 \\
24 \\
25\end{array}$ & $\begin{array}{l}.82 \\
.84 \\
.93 \\
.77 \\
.56\end{array}$ & $\begin{array}{l}.84 \\
7.8 \\
2.8 \\
.60 \\
.54\end{array}$ & $\begin{array}{l}.98 \\
.88 \\
.91 \\
.94 \\
.85\end{array}$ & $\begin{array}{r}.99 \\
.93 \\
.97 \\
1.1 \\
.90\end{array}$ & $\begin{array}{l}1.1 \\
1.1 \\
1.1 \\
1.1 \\
1.1\end{array}$ & $\begin{array}{l}1.1 \\
1.7 \\
1.7 \\
1.1 \\
.98\end{array}$ & $\begin{array}{c}8.5 \\
.89 \\
.86 \\
.97 \\
.97\end{array}$ & $\begin{array}{l}1.1 \\
1.2 \\
1.3 \\
1.5 \\
1.2\end{array}$ & $\begin{array}{l}1.5 \\
1.4 \\
1.4 \\
1.3 \\
1.2\end{array}$ & $\begin{array}{l}2.6 \\
4.2 \\
1.2 \\
1.2 \\
1.0\end{array}$ & $\begin{array}{r}1.2 \\
25 \\
6.2 \\
1.9 \\
1.2\end{array}$ & $\begin{array}{l}1.0 \\
.98 \\
2.0 \\
4.2 \\
1.0\end{array}$ \\
\hline $\begin{array}{l}26 \\
27 \\
28 \\
29 \\
30 \\
31\end{array}$ & $\begin{array}{r}12^{.69} \\
.61 \\
.58 \\
.54 \\
.51\end{array}$ & $\begin{array}{l}.64 \\
.65 \\
.66 \\
.67 \\
.66 \\
.--\end{array}$ & $\begin{array}{l}.81 \\
.98 \\
1.0 \\
1.2 \\
1.1 \\
4.6\end{array}$ & $\begin{array}{c}.86 \\
1.0 \\
10^{.80} \\
9.6 \\
3.2\end{array}$ & \begin{tabular}{l}
1.0 \\
.90 \\
1.1 \\
1.0 \\
\hdashline-0
\end{tabular} & $\begin{array}{l}.93 \\
1.1 \\
1.1 \\
.84 \\
.59 \\
.67\end{array}$ & $\begin{array}{r}2.1 \\
35 \\
6.5 \\
1.0 \\
.94 \\
-\end{array}$ & $\begin{array}{l}1.1 \\
1.0 \\
.98 \\
1.0 \\
.99 \\
.98\end{array}$ & $\begin{array}{r}1.3 \\
1.2 \\
1.3 \\
1.3 \\
1.3 \\
\end{array}$ & $\begin{array}{l}1.2 \\
1.2 \\
1.2 \\
1.1 \\
1.2 \\
1.1\end{array}$ & $\begin{array}{l}9.4 \\
1.3 \\
1.0 \\
.87 \\
.84 \\
.82\end{array}$ & \begin{tabular}{c}
24 \\
1.8 \\
.93 \\
1.5 \\
2.4 \\
\hdashline.-
\end{tabular} \\
\hline $\begin{array}{l}\text { TOTAL } \\
\text { MEAN } \\
\text { MAX } \\
\text { MIN } \\
\text { CFSM } \\
\text { IN. } \\
\text { AC-FT } \\
(++)\end{array}$ & $\begin{array}{r}72.13 \\
2.33 \\
21 \\
.51 \\
.56 \\
.65 \\
143 \\
2.78\end{array}$ & $\begin{array}{r}138.51 \\
4.62 \\
80 \\
.42 \\
1.12 \\
1.25 \\
275 \\
4.21\end{array}$ & $\begin{array}{r}150.15 \\
4.84 \\
62 \\
.74 \\
1.17 \\
1.35 \\
298 \\
4.24\end{array}$ & $\begin{array}{r}57.92 \\
1.87 \\
17 \\
.67 \\
.45 \\
.52 \\
115 \\
1.64\end{array}$ & $\begin{array}{r}34.69 \\
1.20 \\
4.0 \\
.85 \\
.29 \\
.31 \\
69 \\
.33\end{array}$ & $\begin{array}{r}31.39 \\
1.01 \\
1.7 \\
.59 \\
.24 \\
.28 \\
62 \\
.09\end{array}$ & $\begin{array}{r}75.93 \\
2.53 \\
35 \\
.70 \\
.61 \\
.68 \\
151 \\
2.36\end{array}$ & $\begin{array}{r}265.55 \\
8.57 \\
101 \\
.98 \\
2.08 \\
2.39 \\
527 \\
7.11\end{array}$ & $\begin{array}{r}193.7 \\
6.46 \\
132 \\
1.1 \\
1.56 \\
1.74 \\
384 \\
4.37\end{array}$ & $\begin{array}{r}70.7 \\
2.28 \\
14 \\
1.0 \\
.55 \\
.64 \\
140 \\
2.38\end{array}$ & $\begin{array}{r}109.13 \\
3.52 \\
27 \\
.82 \\
.85 \\
.98 \\
216 \\
3.64\end{array}$ & $\begin{array}{r}59.03 \\
1.97 \\
24 \\
.85 \\
.48 \\
.53 \\
117 \\
1.79\end{array}$ \\
\hline $\begin{array}{l}\text { CAL YR } \\
\text { WTR YR }\end{array}$ & $\begin{array}{l}1971 \\
1972\end{array}$ & $\begin{array}{ll}\text { TOTAL } 1,0 \\
\text { TOTAL } 1,2\end{array}$ & $\begin{array}{l}\text { MEAN } \\
\text { MEAN }\end{array}$ & $\begin{array}{l}2.85 \\
3.44\end{array}$ & $\begin{array}{lr}\operatorname{MAX} & 80 \\
\operatorname{MAX} & 132\end{array}$ & $\begin{array}{l}\text { MIN } .42 \\
\text { MIN }: 42\end{array}$ & $\begin{array}{l}\text { CFSM } \\
\text { CFSM : }\end{array}$ & $\begin{array}{l}\text { IN } \\
\text { IN } 1\end{array}$ & $\begin{array}{l}A C-F T \\
A C-F T\end{array}$ & $\begin{array}{l}2,060 \\
2,500\end{array}$ & $\begin{array}{l}t+28.92 \\
+t \quad 34.94\end{array}$ & \\
\hline
\end{tabular}

DATE TIME G.HT. DISCHARGE

$\begin{array}{rrrr}5-2 & 0030 & 6.91 & 2,160 \\ 6-16 & 1600 & 5.10 & 975 \\ 8-22 & 1800 & 4.88 & 860\end{array}$

1/This table reproduced from U.S. Geological Survey, 1972, Water Resources Data for Texas, Part 1, Surface Water Records. 


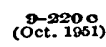

yearly weighted-mean rainfall Monthly and $九$

in

inches
UNITED STATES

DEPARTMENT OF THE INTERIOR

GEOLOGICAL SURVEY

WATER PESOURCES DIVISION
Sheet___ 1 of ______ Sheets

$8-1575$.

of Waller Creek at 23rd St., Austin, Tex.

[Drainage area, -4.13 square miles]

\begin{tabular}{|c|c|c|c|c|c|c|c|c|c|c|c|c|c|c|}
\hline \multicolumn{12}{|c|}{ age at } & \multirow{2}{*}{$\frac{16-26489-5}{\text { SEPT. }}$} & \multicolumn{2}{|c|}{ U. S. GOVERRURKT PRIUTING OOFECE } \\
\hline YEAR & OCT. & Nov. & DEC. & JAN. & FEB. & MAR. & APR. & MAY & JUNE & JULY & AUG. & & ANNUAL & Cal. Yr. \\
\hline 1956 & - & - & - & - & - & 0.42 & 0.58 & 3.08 & 1.04 & 0.43 & 1.38 & 0.21 & - & \\
\hline 1957 & 1.31 & 2.06 & 2.08 & 0.50 & 2.51 & 3.73 & 9.53 & 6.84 & 5.04 & .63 & .02 & 6.70 & 40.95 & 49.99 \\
\hline 1958 & 10.11 & 3.14 & 1.24 & 3.05 & 6.62 & 2.60 & 4.82 & 3.16 & 4.02 & 2.02 & .68 & 7.32 & 48.78 & 42.38 \\
\hline 1959 & 6.01 & .88 & 1.20 & .44 & 2.69 & .22 & 4.80 & 2.04 & 3.41 & 4.79 & 4.70 & 4.04 & 35.22 & 38.23 \\
\hline 1960 & 7.22 & 1.67 & 2.21 & 1.17 & 2.35 & 1.29 & 1.12 & .98 & 4.34 & 2.39 & 3.60 & 1.49 ! & 29.83 & 37.93 \\
\hline 1961 & 12.53 & 2.17 & 4.50 & 1.44 & 4.92 & .67 & .18 & 1.09 & 11.98 & 8.27 & .88 & 3.82 & 52.47 & $38.19^{\circ}$ \\
\hline 1962 & .94 & 2.30 & 1.68 & .65 & .92 & 1.20 & 4.82 & 1.39 & 8.32 & .02 & 3.75 & 4.35 & 30.34 & 33.49 \\
\hline 1963 & 3.83 & 1.12 & 3.12 & .49 & 2.64 & .22 & 2.92 & 1.16 & 3.56 & .07 & .49 & 1.27 & 20.89 & 16.74 \\
\hline 1964 & .86 & 1.70 & 1.36 & 3.00 & 1.56 & 2.14 & 1.46 & 1.75 & 5.52 & .98 & 1.74 & 8.00 & 30.06 & 34.12 \\
\hline 1965 & 4.16 & 2.78 & 1.04 & 4.36 & 5.79 & 1.46 & 1.85 & 9.92 & 1.57 & .32 & .65 & 6.38 & 40.28 & 44.34 \\
\hline 1966 & 3.58 & 3.41 & 5.05 & 1.33 & 2.86 & .62 & 4.06 & 3.52 & .89 & .32 & 7.33 & 3.86 & 36.83 & 26.62 \\
\hline 1967 & 1.02 & .10 & .71 & .17 & 1.43 & .87 & 4.40 & 3.87 & 0 & .80 & 5.09 & 6.32 & 24.78 & 35.62 \\
\hline 1968 & 4.62 & 4.54 & 3.51 & 8.63 & 1.56 & 2.53 & 2.38 & 7.28 & 2.69 & 2.87 & .82 & 2.82 & 44.25 & 38.44 \\
\hline 1969 & .60 & 5.62 & .64 & .34 & 4.08 & 2.78 & 4.97 & 3.03 & 2.18 & .27 & 4.85 & 1.32 & 30.68 & 32.71 \\
\hline 1970 & 3.27 & .83 & 4.79 & 1.92 & 5.85 & 2.81 & 1.24 & 6.91 & .23 & .43 & 1.88 & 4.79 & 34.95 & 31.01 \\
\hline 1971 & 4.86 & 0 & .09 & .04 & .90 & .69 & 1.06 & 1.50 & 2.39 & 1.84 & 6.39 & 2.88 & 22.64 & 28.92 \\
\hline 1972 & 2.78 & 4.21 & 4.24 & 1.64 & .33 & .09 & 2.36 & 7.11 & 4.37 & 2.38 & 3.64 & 1.79 & 34.94 & \\
\hline & & & & & & & & & & & & & & \\
\hline & & & & & & & & & & & & & & \\
\hline & & & & & & & & & & & & & & \\
\hline & & & & & & & & & & & & & & \\
\hline & & & & & & & & & & & & & & \\
\hline & & & & & & & & & & & & & & \\
\hline & & & & & & & & & & & & & & \\
\hline & & & & & & & & & & & & & & \\
\hline
\end{tabular}




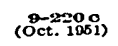

UNITED STATES

DEPARTMENT OF THE INTERIOR

WATER RESOURCES DIVISION

yearly mean $\mathrm{ft}^{3} / \mathrm{s}$, of Waller Creek @ 23rd St

yearly mean
Monthly and

[Drainage area, -4.13 square miles]
GEOLOGICAL SURVEY

Shcet _ 1 of _____ Sheets 8-1575.

Austin, Tex.

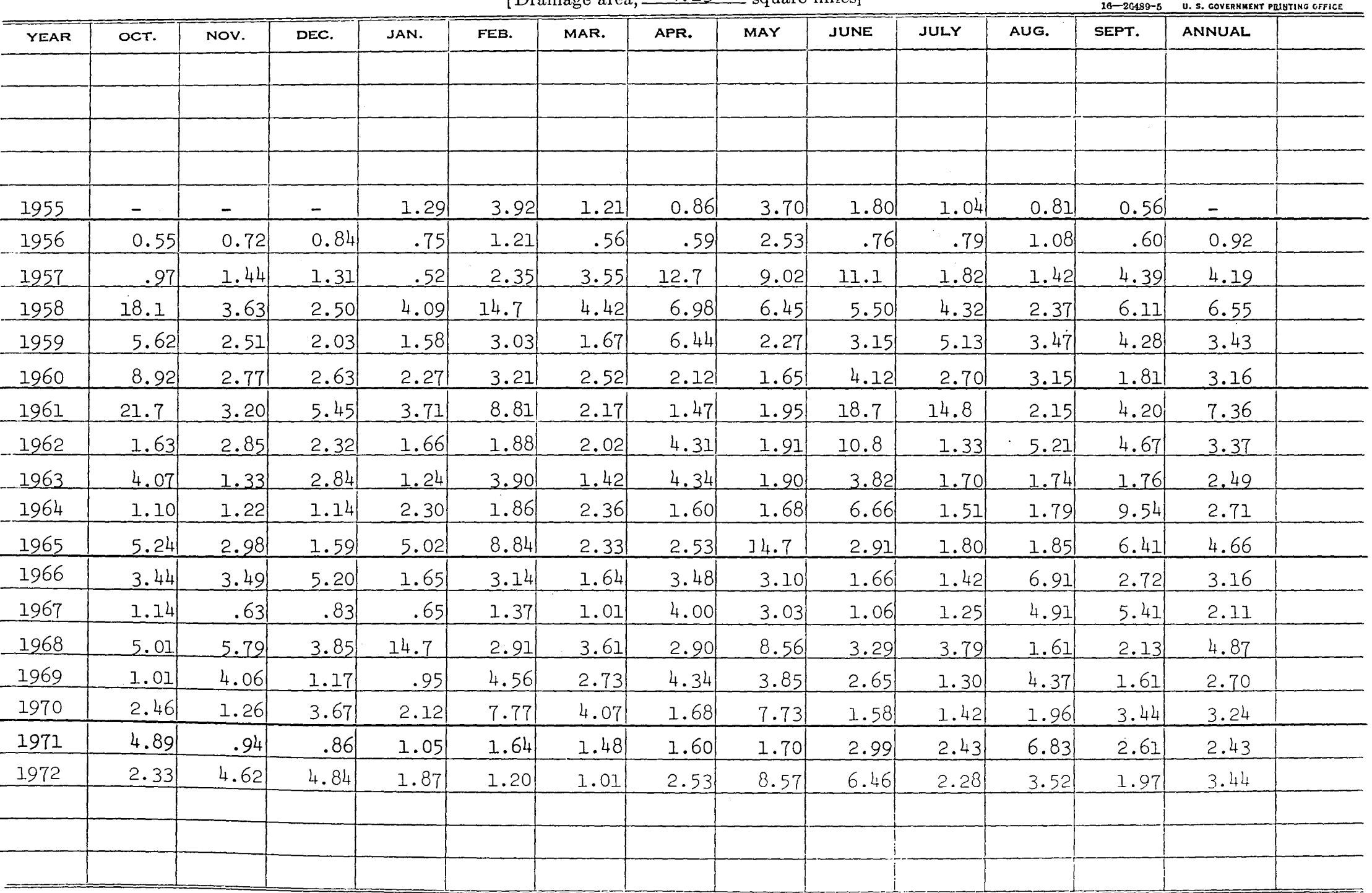


COLORADO RIVER BASIN

08159150 Wilbarger Creek near Pflugerville, Tex. $1 /$

LOCATION.--Lat $30^{\circ} 27^{\prime} 16^{\prime \prime}$, long $97^{\circ} 36^{\prime} 02^{\prime \prime}$, Travis County, on left bank downstream from county road (Pfluger Lane), $800 \mathrm{ft}$ downstream from Farm Road 685, 1.6 miles northeast of Pflugervilile, and 1.9 miles downstream from Missouri-Kansas-Texas Railroad.

DRAINAGE AREA. $--4.61 \mathrm{sq} \mathrm{mi}$.

PERIOD OF RECORD.--August 1963 to current year.

GAGE.--Water-stage recorder and concrete control. Datum of gage is $670.61 \mathrm{ft}$ above mean sea level.

AVERAGE DISCHARGE. --9 years, $1.84 \mathrm{cfs}(5.42$ inches per year, 1,330 acre-ft per year).

EXTREMES.--Current year: Maximum discharge, $563 \mathrm{cfs}$ Nov. 18 (gage height, $4.28 \mathrm{ft}$ ); no flow for many days.

Period of record: Maximum discharge, 1,760 cfs June 16, 1964 (gage height, 6.92 ft); no flow at times each year.

Maximum stage since at least 1894, occurred in September 1921, stage unknown, from information by local residents.

REMARKS.--Records good. Station is part of a hydrologic research project to study rainfall-runoff relations for small urban-rural areas. Three recording rain gages located in watershed above station.

DISCHAKGE, IN CUHIC FEET PER SECONO, WATER YEAR OCTOBER 1971 TO SEPTEMBER 1972

\begin{tabular}{|c|c|c|c|c|c|c|c|c|c|c|c|c|c|}
\hline DAY & 00 & & NOV & $D E C$ & JAN & FEE & MAR & $A P R$ & MAY & JUN & JUL & AUG & SEP \\
\hline $\begin{array}{l}1 \\
2 \\
3 \\
4 \\
5\end{array}$ & & & $\begin{array}{l}0 \\
0 \\
0 \\
0 \\
0\end{array}$ & $\begin{array}{c}.11 \\
4.83 \\
.81 \\
.52 \\
31\end{array}$ & $\begin{array}{l}2.3 \\
1.8 \\
1.8 \\
2.0 \\
1.2\end{array}$ & $\begin{array}{l}2.1 \\
1.8 \\
.99 \\
.88 \\
.91\end{array}$ & $\begin{array}{l}.33 \\
.33 \\
.30 \\
.25 \\
.25\end{array}$ & $\begin{array}{l}.13 \\
.12 \\
.11 \\
.09 \\
.11\end{array}$ & $\begin{array}{r}40.02 \\
.55 \\
.38 \\
.33\end{array}$ & $\begin{array}{l}.21 \\
.21 \\
.21 \\
.21 \\
.17\end{array}$ & $\begin{array}{l}.07 \\
.06 \\
.04 \\
.32 \\
.17\end{array}$ & $\begin{array}{l}.02 \\
.03 \\
.05 \\
.05 \\
.03\end{array}$ & \\
\hline $\begin{array}{r}6 \\
7 \\
8 \\
9 \\
10\end{array}$ & & & $\begin{array}{l}0 \\
0 \\
0 \\
0 \\
0\end{array}$ & $\begin{array}{l}9.0 \\
5.1 \\
3.7 \\
8.3 \\
8.2\end{array}$ & $\begin{array}{l}1.7 \\
1.5 \\
1.5 \\
1.5 \\
1.5\end{array}$ & $\begin{array}{l}1.3 \\
.96 \\
.88 \\
.88 \\
.88\end{array}$ & $\begin{array}{l}.25 \\
.25 \\
.25 \\
.25 \\
.25\end{array}$ & $\begin{array}{l}.11 \\
.10 \\
.07 \\
.06 \\
.06\end{array}$ & $\begin{array}{c}13 \\
3.1 \\
2.2 \\
1.2 \\
7.5\end{array}$ & $\begin{array}{l}.15 \\
.15 \\
.15 \\
.19 \\
.21\end{array}$ & $\begin{array}{l}.10 \\
.09 \\
.08 \\
.08 \\
.08\end{array}$ & $\begin{array}{l}.03 \\
.02 \\
.02 \\
.02 \\
.03\end{array}$ & \\
\hline $\begin{array}{l}11 \\
12 \\
13 \\
14 \\
15\end{array}$ & & & $\begin{array}{l}0 \\
0 \\
0 \\
0 \\
0\end{array}$ & $\begin{array}{l}3.6 \\
2.9 \\
2.6 \\
2.7 \\
2.0\end{array}$ & $\begin{array}{l}1.4 \\
1.2 \\
1.0 \\
.91 \\
.69\end{array}$ & $\begin{array}{l}1.4 \\
1.4 \\
1.0 \\
.89 \\
.84\end{array}$ & $\begin{array}{l}.25 \\
.25 \\
.25 \\
.24 \\
.21\end{array}$ & $\begin{array}{l}.08 \\
.07 \\
.05 \\
.04 \\
.04\end{array}$ & $\begin{array}{l}5.1 \\
5.4 \\
3.6 \\
3.1 \\
2.4\end{array}$ & $\begin{array}{l}.21 \\
.21 \\
.21 \\
.21 \\
.24\end{array}$ & $\begin{array}{l}.08 \\
.08 \\
.07 \\
.05 \\
.04\end{array}$ & $\begin{array}{l}.04 \\
.04 \\
.03 \\
.02 \\
.02\end{array}$ & \\
\hline $\begin{array}{l}16 \\
17 \\
18 \\
19 \\
20\end{array}$ & & & $\begin{array}{l}0 \\
1.1 \\
50 \\
.10 \\
.07\end{array}$ & $\begin{array}{l}1.8 \\
1.6 \\
1.5 \\
1.5 \\
1.4\end{array}$ & $\begin{array}{l}.62 \\
.62 \\
.83 \\
.88 \\
.88\end{array}$ & $\begin{array}{l}.72 \\
.65 \\
.53 \\
.49 \\
.49\end{array}$ & $\begin{array}{l}.20 \\
.18 \\
.17 \\
.15 \\
.20\end{array}$ & $\begin{array}{l}.03 \\
.02 \\
.02 \\
.02 \\
.02\end{array}$ & $\begin{array}{l}2.0 \\
2.7 \\
1.8 \\
1.4 \\
1.1\end{array}$ & $\begin{array}{l}8.4 \\
1.7 \\
.68 \\
.45 \\
.34\end{array}$ & $\begin{array}{l}.04 \\
.04 \\
.04 \\
.06 \\
.08\end{array}$ & $\begin{array}{l}.02 \\
.02 \\
.03 \\
.01 \\
.01\end{array}$ & \\
\hline $\begin{array}{l}21 \\
22 \\
23 \\
24 \\
25\end{array}$ & & & $\begin{array}{l}.06 \\
.10 \\
.96 \\
.15 \\
.15\end{array}$ & $\begin{array}{l}1.2 \\
.98 \\
.98 \\
.98 \\
.98\end{array}$ & $\begin{array}{l}.88 \\
.87 \\
.64 \\
.55 \\
.55\end{array}$ & $\begin{array}{l}.49 \\
.49 \\
.49 \\
.49 \\
.46\end{array}$ & $\begin{array}{l}.25 \\
.21 \\
.21 \\
.20 \\
.18\end{array}$ & $\begin{array}{l}.02 \\
.02 \\
.02 \\
.02 \\
.02\end{array}$ & $\begin{array}{l}.98 \\
.79 \\
.55 \\
.55 \\
.53\end{array}$ & $\begin{array}{l}.25 \\
.23 \\
.18 \\
.18 \\
.17\end{array}$ & $\begin{array}{l}.03 \\
.06 \\
.07 \\
.04 \\
.03\end{array}$ & $\begin{array}{l}0 \\
0 \\
0 \\
.02 \\
.01\end{array}$ & \\
\hline $\begin{array}{l}26 \\
27 \\
28 \\
29 \\
30 \\
31\end{array}$ & & & $\begin{array}{r}.14 \\
.13 \\
.12 \\
.09 \\
.09 \\
-0\end{array}$ & $\begin{array}{r}.98 \\
.98 \\
.98 \\
.98 \\
1.8 \\
1.3\end{array}$ & $\begin{array}{l}.52 \\
.49 \\
.49 \\
3.5 \\
2.9 \\
1.7\end{array}$ & \begin{tabular}{r}
.43 \\
.41 \\
.33 \\
.33 \\
\hdashline-.0 \\
\end{tabular} & $\begin{array}{l}.18 \\
.17 \\
.14 \\
.13 \\
.13 \\
.13\end{array}$ & $\begin{array}{r}.03 \\
.06 \\
.11 \\
.07 \\
.04 \\
\end{array}$ & $\begin{array}{l}.37 \\
.33 \\
.32 \\
.28 \\
.25 \\
.24\end{array}$ & $\begin{array}{r}.13 \\
.13 \\
.12 \\
.10 \\
.08 \\
- \\
\end{array}$ & $\begin{array}{l}.02 \\
.02 \\
.02 \\
.02 \\
.02 \\
.02\end{array}$ & $\begin{array}{l}0 \\
0 \\
0 \\
0 \\
0 \\
0\end{array}$ & - \\
\hline $\begin{array}{l}\text { TOTAL } \\
\text { MEAN } \\
\text { MAX } \\
\text { MIN } \\
\text { CFSM } \\
\text { IN. } \\
\text { AC-FT } \\
(t+)\end{array}$ & 2.6 & $\begin{array}{l}0 \\
0 \\
0 \\
0 \\
0 \\
0 \\
0 \\
8\end{array}$ & $\begin{array}{r}53.26 \\
1.78 \\
50 \\
0 \\
.39 \\
.43 \\
106 \\
4.84\end{array}$ & $\begin{array}{r}105.28 \\
3.40 \\
31 \\
.11 \\
.74 \\
.85 \\
209 \\
3.82\end{array}$ & $\begin{array}{r}38.92 \\
1.26 \\
3.5 \\
.49 \\
.27 \\
.31 \\
77 \\
1.34\end{array}$ & $\begin{array}{r}23.91 \\
.82 \\
2.11 \\
.33 \\
.18 \\
.19 \\
47 \\
.45\end{array}$ & $\begin{array}{r}6.74 \\
.22 \\
.33 \\
.13 \\
.05 \\
.05 \\
13 \\
.44\end{array}$ & $\begin{array}{r}1.76 \\
.059 \\
.13 \\
.02 \\
.01 \\
.01 \\
3.55 \\
.87\end{array}$ & $\begin{array}{r}102.07 \\
3.29 \\
40 \\
.02 \\
.71 \\
.82 \\
202 \\
5.86\end{array}$ & $\begin{array}{r}16.07 \\
.54 \\
8.4 \\
.08 \\
.12 \\
.13 \\
32 \\
2.62\end{array}$ & $\begin{array}{l}2.02 \\
.065 \\
.32 \\
.02 \\
.01 \\
.02 \\
4.00 \\
2.02\end{array}$ & $\begin{array}{r}.57 \\
.018 \\
.05 \\
0 \\
.004 \\
.004 \\
1.81 \\
3.85\end{array}$ & $\begin{array}{r}0 \\
0 \\
0 \\
0 \\
0 \\
0 \\
1.56^{0}\end{array}$ \\
\hline $\begin{array}{l}\text { CAL YR } \\
\text { WTR YR }\end{array}$ & $\begin{array}{l}971 \\
972\end{array}$ & $\begin{array}{l}\text { TOTAL } \\
\text { TOTAL }\end{array}$ & $\begin{array}{l}166.15 \\
350.60\end{array}$ & $\begin{array}{l}\text { MEAN } \\
\text { MEAN }\end{array}$ & $\begin{array}{l}\text { MAX } \\
\text { MAX }\end{array}$ & $\begin{array}{ll}\operatorname{MIN} & 0 \\
\operatorname{MIN} & 0\end{array}$ & $\begin{array}{l}\text { CFSM } \\
\text { CFSM }\end{array}$ & $\begin{array}{l}.10 \\
.21\end{array}$ & $\begin{array}{l}1.34 \\
2.83\end{array}$ & $\begin{array}{ll}\text {-FT } & 330 \\
\text {-FT } & 695\end{array}$ & $\begin{array}{ll}t+ & 24.27 \\
t+ & 30.35\end{array}$ & & \\
\hline
\end{tabular}

PEAK DISCHARGE (BASE, $400 \mathrm{CFS}$ ).--Nov. 18 (0030) $563 \mathrm{cfs}(4.28 \mathrm{ft}$ ); May 2 (0145) $464 \mathrm{cfs}(3.94 \mathrm{ft}$ ).

tt Weighted-mean rainfall, in inches.

1/This table reproduced from U.S. Geological Survey, 1972, Water Resources Data for Texas, Part 1, Surface Water Records. 
(50)

UNITED STATES

DEPARTMENT OF THE INTERIOR

GEOLOGICAL SURVEY

WATER RESOURCES DIVISION

yearly weighted-mean rainfall Monthly and

of Wilbarger Creek

[Drainage area, 4.6] square miles]

\begin{tabular}{|c|c|c|c|c|c|c|c|c|c|c|c|c|c|c|}
\hline & & & & & & age area & 4.61 & squa & iles] & & & $16-26489-5$ & U. 8. GOVERRMENT & PBUSTING CFFICE \\
\hline YEAR & OCT. & Nov. & DEC. & SAN. & FEB. & MAR. & APR. & MAY & JUNE & JULY & AUG. & SEPT. & ANNUAL & Cal. Yr. \\
\hline & & & & & & & & & & & & & & \\
\hline & . & & & & & & & & & & & & & \\
\hline 1964 & 1.33 & 1.85 & 1.44 & 2.86 & 1.43 & 2.24 & 2.87 & 4.70 & 7.43 & 0.23 & 1.59 & 7.52 & 35.49 & 38.01 \\
\hline 1965 & 2.99 & 2.97 & 1.18 & 5.07 & 6.39 & .87 & 1.65 & 5.87 & 2.00 & .23 & 3.23 & 4.97 & 37.42 & 40.75 \\
\hline 1966 & 2.60 & 3.36 & 4.51 & 1.40 & 2.86 & .71 & 5.64 & 2.67 & 1.63 & .15 & 4.95 & 4.00 & 34.48 & 25.43 \\
\hline 1967 & .55 & .10 & .77 & .28 & 1.94 & .85 & 4.01 & 4.60 & 0 & .64 & 2.46 & 4.87 & 21.07 & 32.15 \\
\hline 1968 & 5.13 & 4.10 & 3.27 & 8.17 & 1.63 & 2.32 & 2.62 & 6.55 & 4.00 & 2.36 & 2.37 & 3.20 & 45.72 & 39.32 \\
\hline 1969 & .29 & 4.69 & 1.12 & .32 & 3.97 & $2.80^{\circ}$ & 4.63 & 1.33 & 2.76 & .18 & 4.85 & .98 & 27.92 & 29.98 \\
\hline 1970 & 3.33 & .66 & 4.17 & 1.59 & 5.20 & 3.501 & .951 & 7.58 & .36 & .49 & .63 & 5.21 & 33.67 & 30.89 \\
\hline 1971 & 5.30 & 0 & .08 & 0 & 1.09 & .93 & 1.01 & 1.12 & .95 & 1.23 & 4.95 & 1.65 & 18.31 & 24.27 \\
\hline 1972 & 2.68 & 4.84 & 3.82 & 1.34 & .45 & .44 & .87 & 5.86 & 2.62 & 2.02 & 3.85 & 1.56 & 30.35 & \\
\hline & & & & - & & & & & & & & & & \\
\hline & & & & & & & & & & & & & & \\
\hline & & & & & & & & & & & & & & \\
\hline & & & & & & & & & & & & & & \\
\hline & & & & & & & & & & & & & & \\
\hline & & & & & & & & & & & & & & \\
\hline & & & & & & & & & & & & & & \\
\hline & & & & & & & & & & & & & & \\
\hline & & & & & & & & & & & & & & \\
\hline & & & & & & & & & & & & & & \\
\hline & & & & & & & & & & & & & & \\
\hline & & & & & & & & & & & & & & \\
\hline & & & & & & & & & & & & & & \\
\hline
\end{tabular}

Shect I of I_ Sheets

$8-1591.5$

Pflugerville, Tex. 
yearly mean

Monthly and discharge, in f... $\mathrm{ft} / \mathrm{s}$
GEOLOGICAL SURVEY

Creek

of Wilbarger Cree

[Drainage area, 4.61__ square miles]
$8-1591.5$

Pflugerville, Tex.

\begin{tabular}{|c|c|c|c|c|c|c|c|c|c|c|c|c|c|c|}
\hline YEAR & OCT. & NOV. & DEC. & JAN. & FEB. & MAR. & APR. & MAY & JUNE & JULY & AUG. & SEPT. & ANNUAL & \\
\hline & & & & & & & & & & & & & & \\
\hline 1963 & - & - & - & - & - & - & - & - & - & _ & 0 & 0 & _ & \\
\hline 1064 & 0 & 0 & 0 & 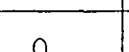 & 0 & 0.03 & 767 & 423 & 104 & 007 & 0 & 202 & 753 & \\
\hline 1904 & $u$ & 0 & 0 & 0 & 0 & 0.03 & 1.01 & 4.23 & 10.4 & 0.01 & 0 & c. $.0<$ & 1.30 & \\
\hline 1965 & .43 & 1.62 & .71 & 6.45 & 15.4 & 2.01 & .75 & 3.32 & .57 & .05 & .08 & .72 & 2.59 & \\
\hline 1966 & .13 & 1.50 & 7.36 & 1.88 & 3.73 & 2.01 & 5.90 & 3.57 & .18 & .03 & .18 & .79 & 2.26 & \\
\hline 1967 & .26 & .10 & .12 & .091 & .15 & .043 & .30 & 1.87 & .042 & 0 & .0003 & .026 & .25 & \\
\hline 1968 & .97 & 6.03 & 5.69 & 16.7 & 3.80 & 4.63 & 3.15 & 8.59 & 2.27 & 1.18 & .078 & .085 & 4.45 & \\
\hline 1969 & .020 & .16 & .46 & .22 & 3.74 & 5.43 & 7.07 & .89 & .15 & . 011 & .019 & .012 & 1.49 & \\
\hline 1970 & .006 & .003 & .51 & 1.26 & 9.53 & 11.4 & 1.07 & 8.94 & 1.37 & .058 & .002 & .013 & 2.82 & \\
\hline 1971 & 2.29 & .26 & .15 & .095 & .068 & .059 & .029 & 0 & 0 & 0 & .002 & 0 & .25 & \\
\hline 1972 & 0 & 1.78 & 3.40 & 1.26 & .82 & .22 & .059 & 3.29 & .54 & .065 & .018 & 0 & .96 & \\
\hline & & & & & & & & & & & & & & \\
\hline & & & & & & & & & & & & & & \\
\hline & & & & & & & & & & & & & & \\
\hline & & & & & & & & & & & & & & \\
\hline & & & & & & & & & & & & & & \\
\hline & & & & & & & & & & & & & & \\
\hline & & & & & & & & & & & & & & \\
\hline & & & & & & & & & & & & & & \\
\hline & & & & & & & & & & & & & & \\
\hline & & & & & & & & & & & & & & \\
\hline & & & & & & & & & & & & & & \\
\hline & & & & & & & & & & & & & & \\
\hline & & & & & & & & & & & & & & \\
\hline
\end{tabular}


STUDY AREA WALLER CREEK

\begin{tabular}{|l|l|l|l|l|l|}
\hline Date of storm $1-5$ & $2-5$ & $3-5$ & $4-R$ & $5-R$ & $6-R$ \\
\hline
\end{tabular} \begin{tabular}{l|l|l|l|l|l}
.264 & .101 & .190 & .161 & .187 & .097
\end{tabular} $.360 .006 .338 .287 .009=$ $.264 .101-.351 .187 .097$ $.360 .006-.625 .009=$ $-.232-.378 .232 .158$
RAINFALL DATA SUMMARY

RAIN GAGE

\section{*} \begin{tabular}{|l|l|l|l|l} 
W.M.R. WR \\
$23 R O$ & $38+H$ \\
\hline
\end{tabular} \begin{tabular}{l|l|l|} 
W.M.R. W.M.R. \\
23 RO & $38 T^{4}$ \\
\hline
\end{tabular} 23 Rㅇ 38 TH

1972 WATER YEAR

\begin{tabular}{|c|c|c|c|c|c|c|c|c|c|c|c|c|c|c|c|c|c|c|c|c|}
\hline & .264 & .101 & .190 & .161 & .187 & .097 & $=$ & 3 & $30-$ & & $\square$ & 1 & & \begin{tabular}{|l|l|} 
& \\
\end{tabular} & \begin{tabular}{|l|l|} 
& \\
\end{tabular} & 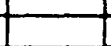 & & & & \\
\hline & .360 & .006 & .338 & 287 & .009 & & & & $E$ & & & & & & & & & & & \\
\hline & & & & & & & & & & & & & & & & & & & & \\
\hline & .264 & .101 & $=$ & .351 & .187 & .097 & & & & & $=$ & & & W & & & & & & \\
\hline & .360 & .006 & $=$ & .625 & .009 & $=$ & & & & & & $\Xi$ & & & & & & & & \\
\hline & & & & & & & & & & & & & & & & & & & & \\
\hline & - & .232 & $=$ & 378 & .232 & .158 & & & & & & & & 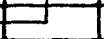 & & & & & & \\
\hline & $=$ & .259 & $\overline{-}$ & 574 & 167 & & & & & & & & & & $\Xi$ & & & & & \\
\hline $3-4$ & .75 & .75 & & .82 & .62 & .57 & & & & & & & & & & & & & & \\
\hline 5 & .01 & .02 & 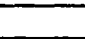 & - & .01 & - & & & & & & & & & & & & & & \\
\hline 8 & $=$ & $T$ & & .08 & .09 & .07 & & & & & & & & & & & & & & \\
\hline $8-9$ & $=$ & .25 & & .21 & $=$ & $=$ & & & & & & & & & & & & & & \\
\hline 9 & .41 & & & $=1$ & $(.50)$ & .46 & & & & & & & & & & & & & 8 & \\
\hline 14 & .06 & $=$ & & .02 & - & $=$ & & & & & & & & & & & & & & \\
\hline 17 & .15 & $T$ & & .10 & .04 & .05 & & & & & & & & & & & & & & \\
\hline 18 & .08 & .12 & & .05 & .03 & .05 & & & & & & & & & & & & & & \\
\hline 19 & - & .05 & & - & - & .08 & & & & & & & & & & & & & & \\
\hline $19-20$ & .96 & .75 & & .74 & .91 & 1.00 & & & & & & & & & & & & & & \\
\hline 23 & .05 & - & & .03 & E & $=$ & & & & & & & & & & & & & & \\
\hline $26-27$ & .62 & .56 & & .61 & $=$ & $=$ & & & & & & & & & & & & & & \\
\hline 27 & $T$ & - & & - & .50 & .57 & & & & & & & & & & & & & & \\
\hline QCT. TOTALS & 3.09 & 2.50 & & 2.66 & 2,70 & 2.85 & & & & & 2.78 & 2.81 & & & & & & & & \\
\hline & & & & & & & & & & & & & & & & & & & & \\
\hline Nov. & & $T$ & & .05 & $=$ & - & & & & & & & & & & & & & & \\
\hline $17-18$ & & 4.00 & & 3.90 & 3.50 & 2.92 & & & & & & & & & & & & & & \\
\hline 18 & & - & & $=$ & .04 & $=$ & & & & & & & & & & & & & & \\
\hline 22 & & .57 & & .53 & .37 & .37 & & & & & & & & & & & & & & \\
\hline 30 & & $T$ & & .04 & .05 & .02 & & & & & & & & & & & & & & \\
\hline NOV. TOTALS & & 4.57 & & 4.52 & 3.96 & 3.31 & & & & & & & & 4.21 & 4.44 & & & & & \\
\hline & & & & & & & & & & & & & & & & & & & & \\
\hline DEC. & & .85 & & 1,36 & 1.52 & .81 & & & & & & & & & & & & & & \\
\hline 2 & & .62 & & $=$ & - & .66 & & & & & & & & & & & & & & \\
\hline $4-5$ & & 1.62 & & $(1.66)$ & 1.51 & 1.47 & & & & & & & & & & & & & & \\
\hline $5-6$ & & .14 & & $(.14)$ & .17 & .20 & & & & & & & & & & & & & & \\
\hline 6 & & .10 & & .04 & .09 & .06 & & & & & & & & & & & & & & \\
\hline 8 & & .32 & & .24 & .23 & $(.27)$ & & & & & & & & & & & & & & \\
\hline 9 & & .36 & & .38 & .27 & $(.31)$ & & & & & & & & & & & & & & \\
\hline$\pi$ & & $T$ & & .04 & .04 & .02 & & & & & & & & & & & & & & \\
\hline 12 & & .05 & & .05 & .05 & .01 & & & & & & & & & & & & & & \\
\hline 13 & & $T$ & & .02 & .02 & .01 & & & & & & & & & & & & & & \\
\hline
\end{tabular}




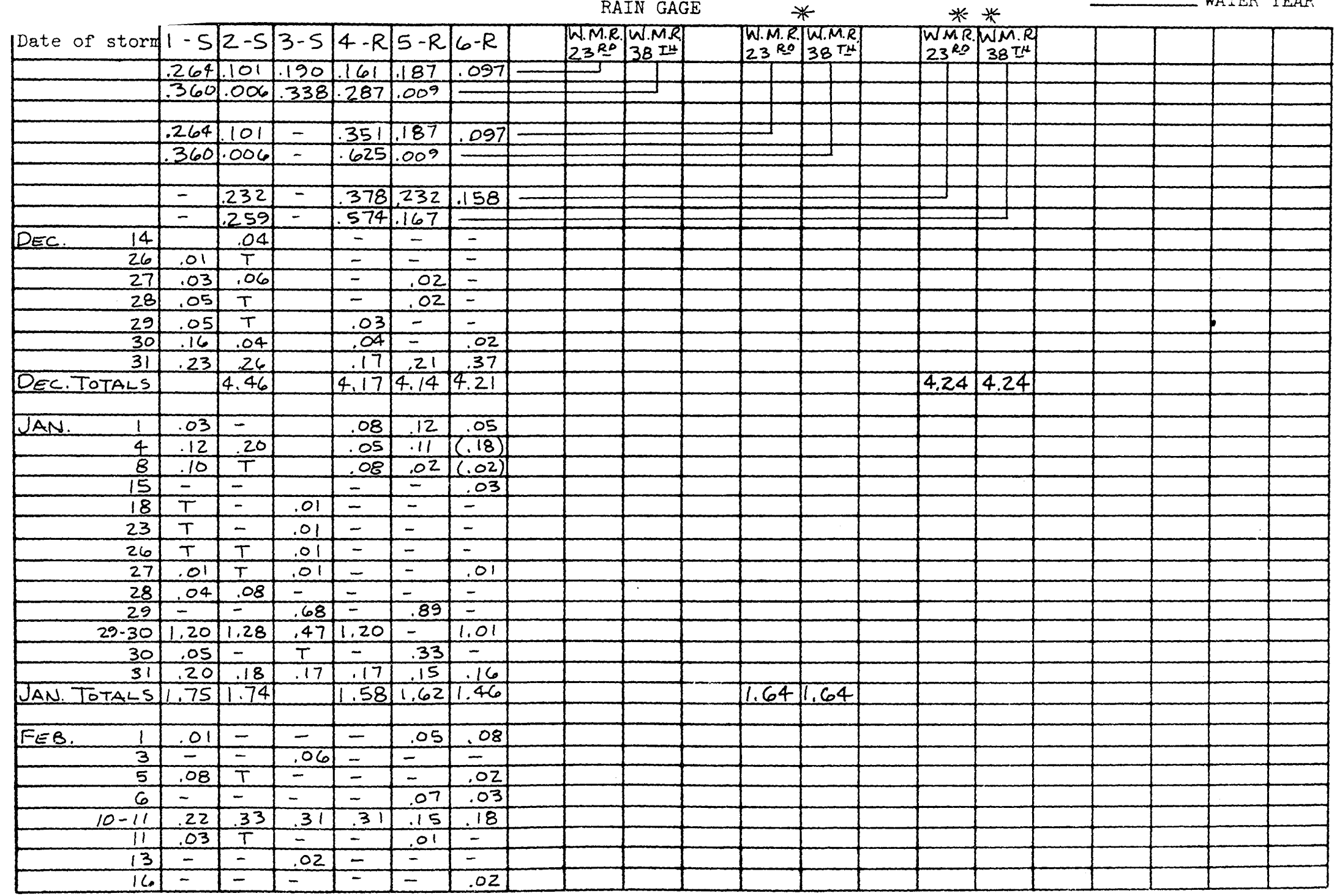


study area Waller CReEK

\begin{tabular}{|c|c|c|c|c|c|c|c|}
\hline Date of storm & $-s$ & $2-5$ & $3-5$ & $4-R$ & $5-R$ & $6-R$ & \\
\hline & 264 & 101 & .190 & .161 & .187 & .097 & \\
\hline & .360 & .006 & 338 & .287 & .009 & & \\
\hline & 264 & 101 & - & 351 & 187 & 097 & \\
\hline & .360 & .006 & - & .625 & 009 & & \\
\hline & & & & & & & \\
\hline & - & 232 & - & 378 & 232 & 158 & \\
\hline & -5 & 259 & - & .574 & .67 & & \\
\hline$E=B$. & $=$ & $I$ & $=$ & $=$ & - & $=$ & \\
\hline & $T$ & $T$ & $=$ & - & - & & \\
\hline FEB. TOTALS & .34 & .33 & .39 & .31 & .28 & .33 & \\
\hline MAPH & & & & & - & & \\
\hline$\frac{10}{20}$ & .06 & $\frac{T}{10}$ & .12 & .14 & .07 & $\frac{-}{.02}$ & \\
\hline ARCH TOTALS & .07 & .10 & 12 & .14 & .07 & .02 & \\
\hline PRIL & 47 & 52 & 48 & 54 & 46 & $(47)$ & \\
\hline$\frac{21}{26}$ & $\frac{41}{. T}$ & $\frac{32}{25}$ & $\frac{.40}{25}$ & .24 & .25 & $\frac{1.41}{.15}$ & \\
\hline & .62 & 1.61 & .26 & 1.23 & 1.35 & 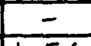 & \\
\hline $27-28$ & -1 & 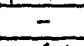 & - & & & 1.56 & \\
\hline 28 & 28 & .41 & .31 & .14 & 37 & & \\
\hline PRIL Totals & 2.37 & 2.79 & 2.30 & 2.15 & 2.43 & 2.18 & \\
\hline MaY & 3.16 & 3.20 & 2.56 & 2.55 & 2.85 & 2.31 & \\
\hline & .24 & 1.08 & 73 & .63 & .65 & .51 & \\
\hline & 89 & .02 & .97 & 46 & 50 & 40 & \\
\hline$\frac{6-7}{7}$ & 80 & .06 & .11 & 57 & .80 & - & \\
\hline 7 & 7 & 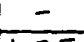 & - & - & - & 2.84 & \\
\hline 10 & 1.27 & 1.25 & 1.20 & 1.30 & 1.31 & $(1.18$ & \\
\hline 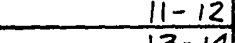 & .15 & .12 & 11 & .10 & .14 & 10) & \\
\hline 13-14 & .87 & .65 & 44 & 51 & 92 & .33 & \\
\hline & 30 & 34 & 27 & 25 & .27 & $(31)$ & \\
\hline NAY TOTALS & 7.68 & 6.72 & 6,39 & 6.37 & 7.44 & 7.98 & \\
\hline UNE & $\Omega, 22$ & 04 & 02 & 15 & 15 & .11 & \\
\hline $9 P M$ & & 20 & E & - & .04 & .07 & \\
\hline $9-10$ & $=$ & $\pi$ & - & 5 & & .10 & \\
\hline 10 & $=$ & 08 & & .05 & 3.17 & - & \\
\hline$\frac{13}{-16}$ & .16 & $\frac{.07}{.97}$ & $\frac{.08}{7.35}$ & $\frac{.06}{3.89}$ & & $\frac{.05}{2.44}$ & \\
\hline
\end{tabular}

RAINFALL DATA SUMMARY

RAIN GAGE

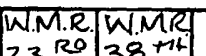

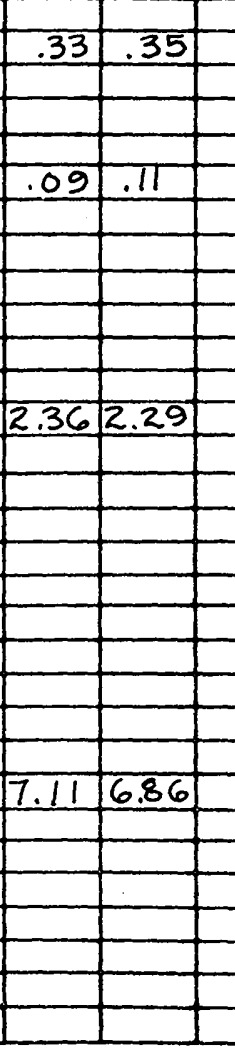

1972 WATER YEAR

* *

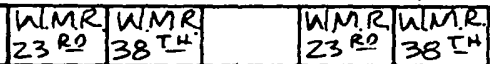

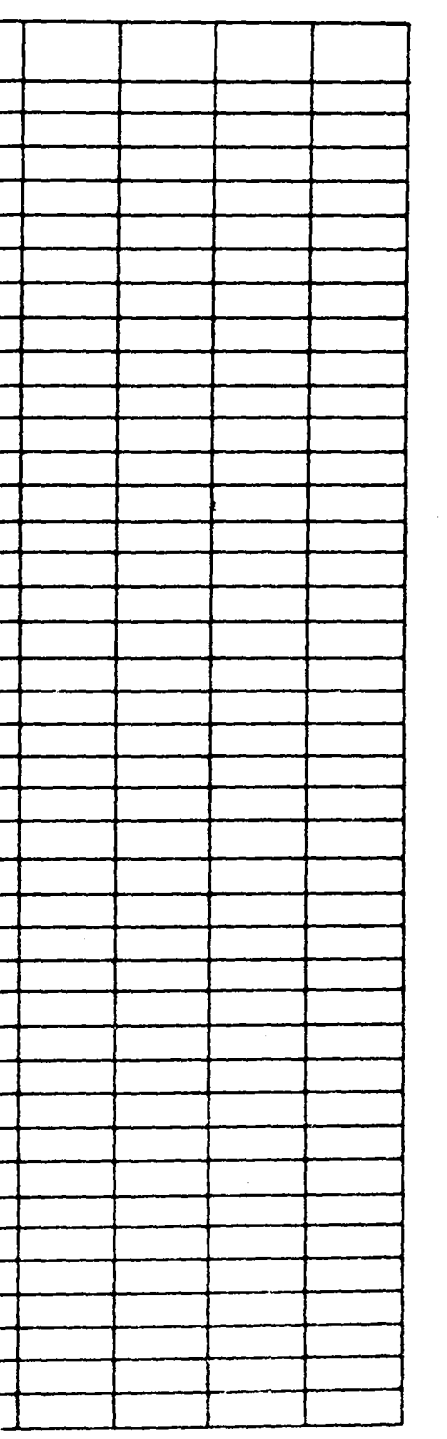


Form TX-88

$10-69$
UNITED STATES DEPARTMENT OF THE INTERIOR

GEOLOGICAL SURVEY-TEXAS DISTRICT study area Waller Creek

\begin{tabular}{|c|c|c|c|c|c|c|}
\hline te & -5 & $2-s$ & & $=R$ & 5-R & $6^{-}$ \\
\hline & .264 & .101 & .190 & .161 & 187 & \\
\hline & .360 & .006 & .338 & .287 & .009 & \\
\hline & 764 & 101 & & 351 & 27 & \\
\hline & 360 & .006 & & .625 & 1009 & \\
\hline & & & & & & \\
\hline & E & .232 & & .378 & $\sqrt{.232}$ & \\
\hline & $\equiv$ & 259 & & 574 & 167 & \\
\hline JNE & .14 & .28 & 02 & .03 & .16 & \\
\hline UNE TOTALS & 3.27 & 4.64 & 7.47 & 4.18 & 3.52 & \\
\hline ULY & .63 & 75 & 77 & 75 & 64 & \\
\hline 는 & 15 & 12 & 39 & 12 & .05 & \\
\hline & II & I & & & $=$ & \\
\hline 10 & $T$ & - & .08 & 50 & .01 & \\
\hline 17 & .10 & .10 & .18 & 11 & $(.09$ & \\
\hline IBAM & .06 & $T$ & - & $=$ & - & \\
\hline $18 P M$ & & .40 & .40 & 17 & 1.07 & \\
\hline 19 & .94 & $\frac{74}{02}$ & 40 & $\frac{58}{13}$ & $\frac{40}{13}$ & \\
\hline & .24 & $\frac{.08}{.24}$ & $\bar{E}$ & 03 & $\frac{13}{45}$ & \\
\hline & & & & & & \\
\hline
\end{tabular}

\begin{tabular}{|l|l|l|l|l|l|l|l|}
\hline JULY TOTALS & 3.35 & 2.43 & 2.22 & 2.26 & 1.84 & 1.21 \\
\hline
\end{tabular}
RAINFALL DATA SUMMARY

RAIN GAGE

W.M.R. $\begin{aligned} & \text { WI.MR: } \\ & 23 \text { Ro. } \\ & 38 T H\end{aligned}$ $+1$

\section{*} 23 R.

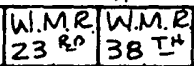

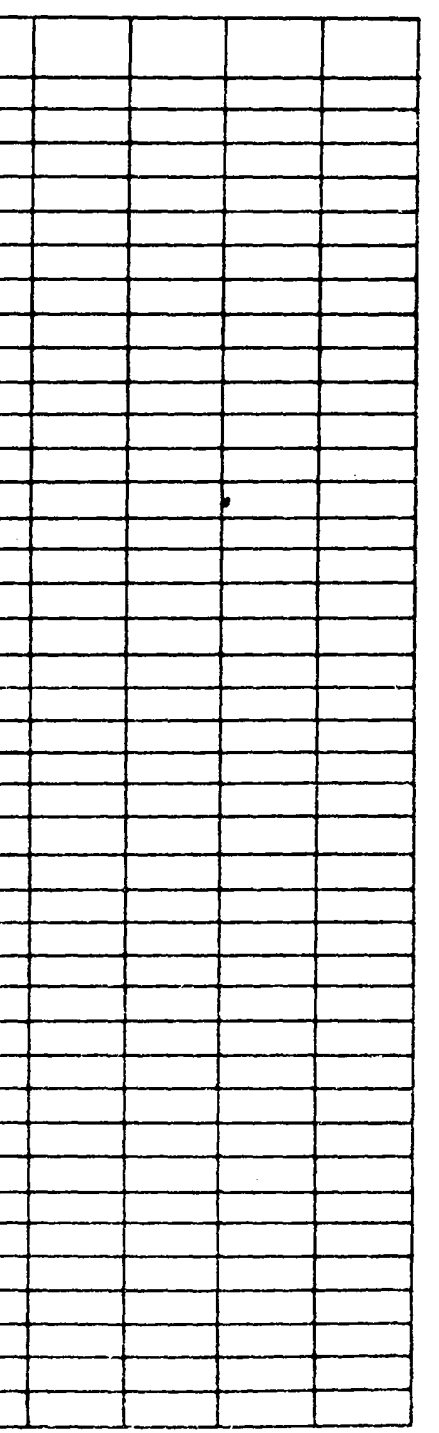


Form TX-88

10-69
UNITED STATES DEPARTMENT OF THE INTERIOR

GEOLOGICAL SURVEY-TEXAS DISTRICT

StUdy area Waller CReEk

RAINFALL DATA SUMMARY

\section{.}

\begin{tabular}{|c|c|c|c|c|c|c|c|c|c|c|c|c|}
\hline Date of storm & $1-5$ & $|2-5|$ & $3-5$ & $4-R$ & $5-R$ & $6-R$ & & 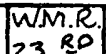 & {$\left[\begin{array}{l}\text { WM.R } \\
3 R^{T}=4\end{array}\right]$} & & 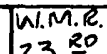 & WMR \\
\hline & 264 & .101 & .190 & .161 & .187 & .097 & & 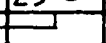 & & & & \\
\hline & 360 & .006 & .338 & 287 & .009 & & & & $E$ & & & \\
\hline & & & & & & & & & & & & \\
\hline & .264 & .101 & & .351 & .187 & .097 & - & & & & E & \\
\hline & .360 & .006 & & .625 & 009 & $\bar{E}$ & & & & & & $\Xi$ \\
\hline & & & & & & & & & & & & \\
\hline & $=$ & .232 & & 378 & 232 & .158 & & & & & & \\
\hline & $=$ & .259 & & .574 & 167 & & & & & $E$ & & \\
\hline SEPT. & $=$ & .04 & = & $=$ & = & $=$ & & & & & & \\
\hline 21 & $=$ & .05 & .06 & - & $=$ & - & & & & & & \\
\hline 23 & .36 & .45 & .42 & .12 & .20 & .15 & & & & & & \\
\hline $24 \mathrm{AM}$ & .06 & .17 & 02 & .28 & .31 & .22 & & & & & & \\
\hline $24 P M$ & - & - & $=$ & $=$ & .13 & - & & & & & & \\
\hline 26 & 1.17 & 1.28 & .73 & 1.08 & 1.14 & 1.12 & & & & & & \\
\hline & .12 & .12 & .05 & $(.10)$ & .13 & 10 & & & & & & \\
\hline SEPT. TOTALS & 1.71 & 2.11 & 1.62 & 1.83 & 1.95 & 1.59 & & 1.79 & 1.72 & & & \\
\hline & & & & & & & & & & & & \\
\hline & & & & & & & & & & & & \\
\hline WATER YEAR & & & & & & & & & & & & \\
\hline Totals & & 36.39 & & 33.49 & 33.87 & 32.57 & & 34.94 & 35.41 & & & \\
\hline & & & & & & & & & & & & \\
\hline & & & & & & & & & & & & \\
\hline & & & & & & & & & & & & \\
\hline & & & & & & & & & & & & \\
\hline & & & & & & & & & & & & \\
\hline & & & & & & & & & & & & \\
\hline & & & & & & & & & & & & \\
\hline & & & & & & & & & & & & \\
\hline & & & & & & & & & & & & \\
\hline & & & & & & & & & & & & \\
\hline & & & & & & & & & & & & \\
\hline & & & & & & & & & & & & \\
\hline & & & & & & & & & & & & \\
\hline & & & & & & & & & & & & \\
\hline & & & & & & & & & & & & \\
\hline & & & & & & & & & & & & \\
\hline
\end{tabular}

RAIN GAGE

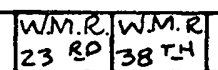


UNITED STATES DEMARMIENT OF THE TNTEHIOR

GEOLOGICAL SURVEY - TEXAS DISTRICT

STUEi ARET WILBARGER CREEK

RAINFALL DATA SUMMMARY

FAIN GAGES

1972 WATER YEAR

\begin{tabular}{|c|c|c|c|c|c|c|c|c|c|c|c|c|c|c|c|c|c|}
\hline Daie of storm & $\frac{1-R}{43}$ & $\frac{2-R}{3 R I}$ & $\frac{3-R}{.268}$ & $n ! M R$ & $\mid \frac{B y}{8+B_{\varepsilon E w}}$ & Date & $\left|\frac{1-R}{431}\right|$ & $-\frac{2-R}{301}$ & $\frac{3-R}{26}$ & WMR & & & $\mid \frac{1-R}{431}$ & $\frac{2-R}{301}$ & $\frac{3-R}{268}$ & |bl.Me & 870 eat \\
\hline OCtober $3-4$ & .97 & .90 & & & & JANUARY & -1 & .95 & - & & 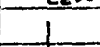 & June & - & .05 & L & & \\
\hline 8 & .07 & .08 & & & & $29-30$ & 1.06 & - & .51 & & & June Totals & 2.08 & 2.71 & 3.39 & 2.62 & \\
\hline 9 & .14 & .15 & & & & 30 & - & .05 & .06 & & & & & & & & \\
\hline 16 & .05 & $=$ & & & & Jan . Totals & 1.45 & 1.39 & 1.12 & 1.34 & & JULY & 1.10 & 1.38 & 1.22 & & \\
\hline $17 A M$ & .17 & .15 & & & & & & & & & & 8 & .02 & .04 & .06 & & \\
\hline $\mid 7 P M$ & .19 & .25 & & & & FEBRUARY 1 & .16 & .11 & .13 & & & 17 & .07 & .35 & .07 & & \\
\hline 19 & .37 & .02 & & & & 4 & $=$ & - & .05 & & & 19 & .23 & .51 & .43 & & \\
\hline $19-20$ & .33 & .42 & .30 & & & $10-11$ & $(.25)$ & .30 & $=$ & & & 21 & $(.10)$ & .14 & .13 & & \\
\hline $26-27$ & .52 & .57 & .53 & & & II & $=$ & - & .32 & & & 22 & $(.05)$ & .30 & .05 & & \\
\hline OCT. TOTALS & 2.81 & 2.54 & * & 2.68 & & 12) & $=$ & $=$ & .05 & & & JULY TOTALS & 1.57 & 2.72 & 1.96 & 2.02 & \\
\hline & & & & & & FEB. TOTALS & .41 & .41 & .55 & 45 & & & & & & & \\
\hline NOVEMBER $17-18$ & 4.26 & 4.17 & & & & & & & & & & AUGUST & $(.05)$ & .05 & 09 & & \\
\hline & .55 & .60 & & & & MARCH & .60 & .39 & 24 & & & 3 & 1.46 & 1.09 & 80 & & \\
\hline 30 & .06 & .03 & & & & MARCH TOTALS & .60 & .39 & .24 & 44 & & 10 & .92 & .60 & 24 & & \\
\hline Nov. TOTALS & 4.87 & 4.80 & * & 484 & & & & & & & & 12 & .10 & .07 & $(.10)$ & & \\
\hline & & & & & & APRIS & .18 & .08 & $=$ & & & 17 & 1.14 & .62 & 49 & & \\
\hline DECEMBER $\perp-2$ & .73 & .70 & & & & 27 & .83 & .73 & 24 & & & 22 & .18 & .05 & 12 & & \\
\hline 2AM-PM & .67 & .58 & & & & 28 & .15 & .10 & .07 & & & 23 & .83 & 1.17 & 43 & & \\
\hline $2 P M$ & .05 & $=$ & & & & 29 & $=$ & - & .05 & & & 29 & .16 & .08 & 11 & & \\
\hline 5 & 1.23 & 1.00 & & & & APRIL TOTALS & 1.16 & .91 & .36 & 87 & & AUG. TOTALS & 4.84 & 3.73 & 2.38 & 3.85 & \\
\hline $6 \mathrm{AM}$ & .10 & .11 & & & & & & & & & & & & & & & \\
\hline GAM-PM: & 23 & .19 & & & & MAY & 2.72 & 2.56 & 2.83 & & & SEPTEMBER 5 & .22 & .20 & .07 & & \\
\hline 8 & 10 & .04 & & & & 5 & $=$ & $=$ & .27 & & & 15 & .13 & .42 & .08 & & \\
\hline SAM & . 35 & 19 & & & & $5-6$ & 45 & .33 & - & & & 16 & .12 & .22 & .07 & & \\
\hline SPM & $L$ & .28 & & & & 6 & .02 & .91 & .75 & & & 21 & .50 & .55 & .20 & & \\
\hline 13 & .06 & - & & & & 7 AM & .32 & .56 & .29 & & & 23 & .48 & .11 & .34 & & \\
\hline 30 & $(.40)$ & .35 & & & & $I P M$ & .10 & - & .09 & & & 26 & .12 & .32 & .09 & & \\
\hline $3 \mid A M-P M$ & $(.05)$ & .05 & .13 & & & 8 & {$[.91$} & .02 & .03 & & & 29 & 10 & .03 & .19 & & \\
\hline $31 P M$ & $(.10)$ & .07 & 12 & & & 10 & $E$ & .95 & .92 & & & SEPT TOTALS & 1.67 & 1.85 & 1.04 & 1.56 & \\
\hline DEC: Totals & 4.07 & 3.56 & * & 3.82 & & $11-12$ & .13 & .10 & .15 & & & & $-2-1$ & & & & \\
\hline & & & & & & $13-14$ & .15 & .12 & .21 & & & & & & & & \\
\hline JANUARY $\perp A M$ & $(.05)$ & .03 & - & & & 16 & $(.20)$ & .20 & .22 & & & WATER YEAR & & & & & \\
\hline $1 P M$ & $-i$ & .04 & .05 & & & MAY TOTALS & 6.00 & 5.75 & 5.76 & 5.86 & & TOTAL & 31.53 & 30.76 & $=$ & 30.35 & end \\
\hline 3 & -.14 & .24 & $\therefore 0$ & & & & & & & & & & $\ldots$ & & & & \\
\hline$\frac{4}{5}$ & $(.03)$ & .04 & -.04 & & & JuNE $12-13$ & & -.10 & $\therefore 17$ & & & & & & & & \\
\hline$-\frac{5}{6}$ & $\frac{(.13)}{-1}$ & -04 & .031 & & & 14 & .90 & .05 & $\frac{.20}{.22}$ & & & & & & & & \\
\hline 16 & E & $=$ & .11 & & & $15-16$ & L & .98 & .21 & & & & & & & & \\
\hline 26 & .04 & - & - & & Eew & 16 & 118 & 1.43 & $\sqrt{2} .59$ & & BTE & & & & & & \\
\hline
\end{tabular}

* ${ }^{*}$ jRECORO LOST AT $3-R$ 


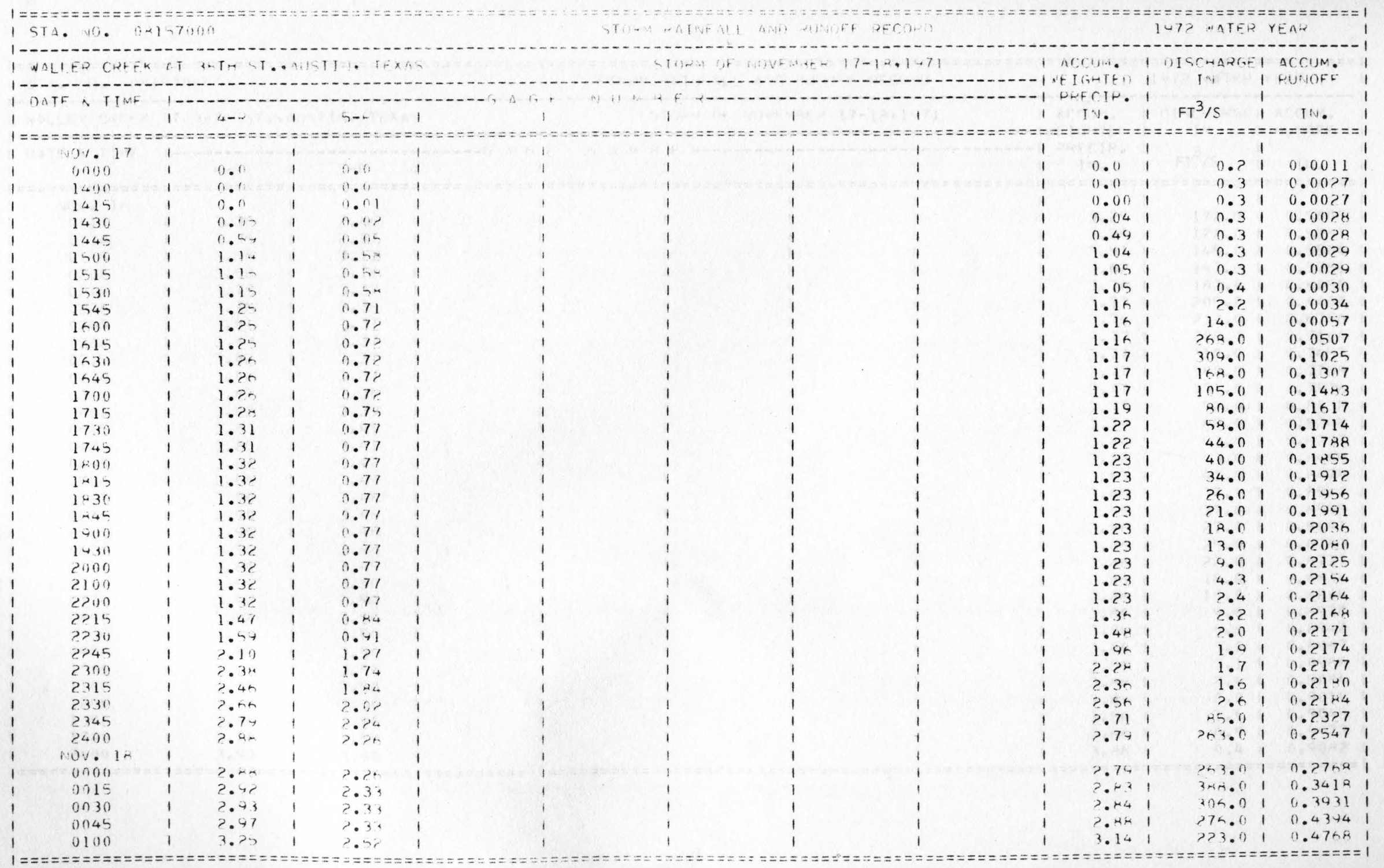




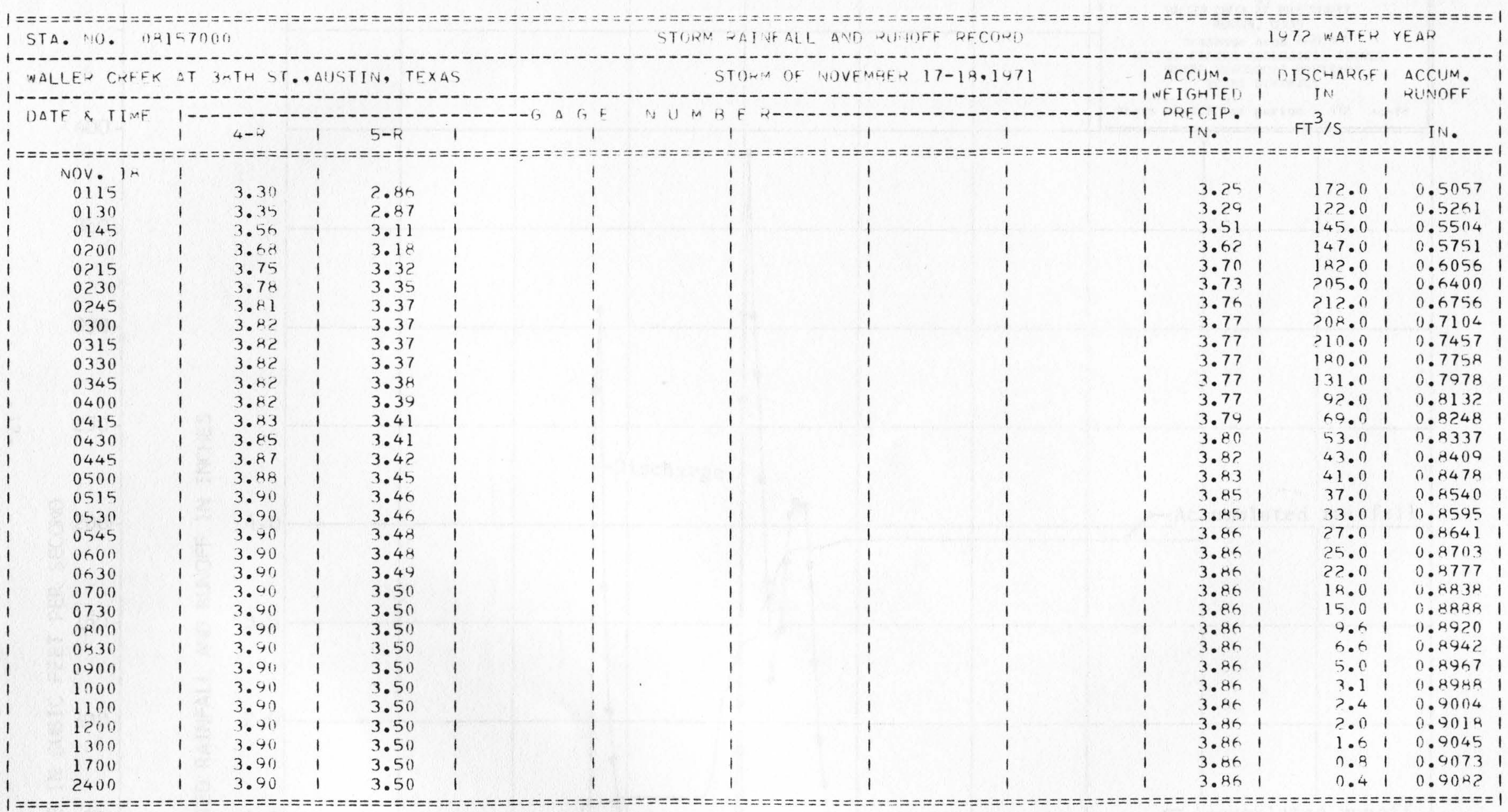




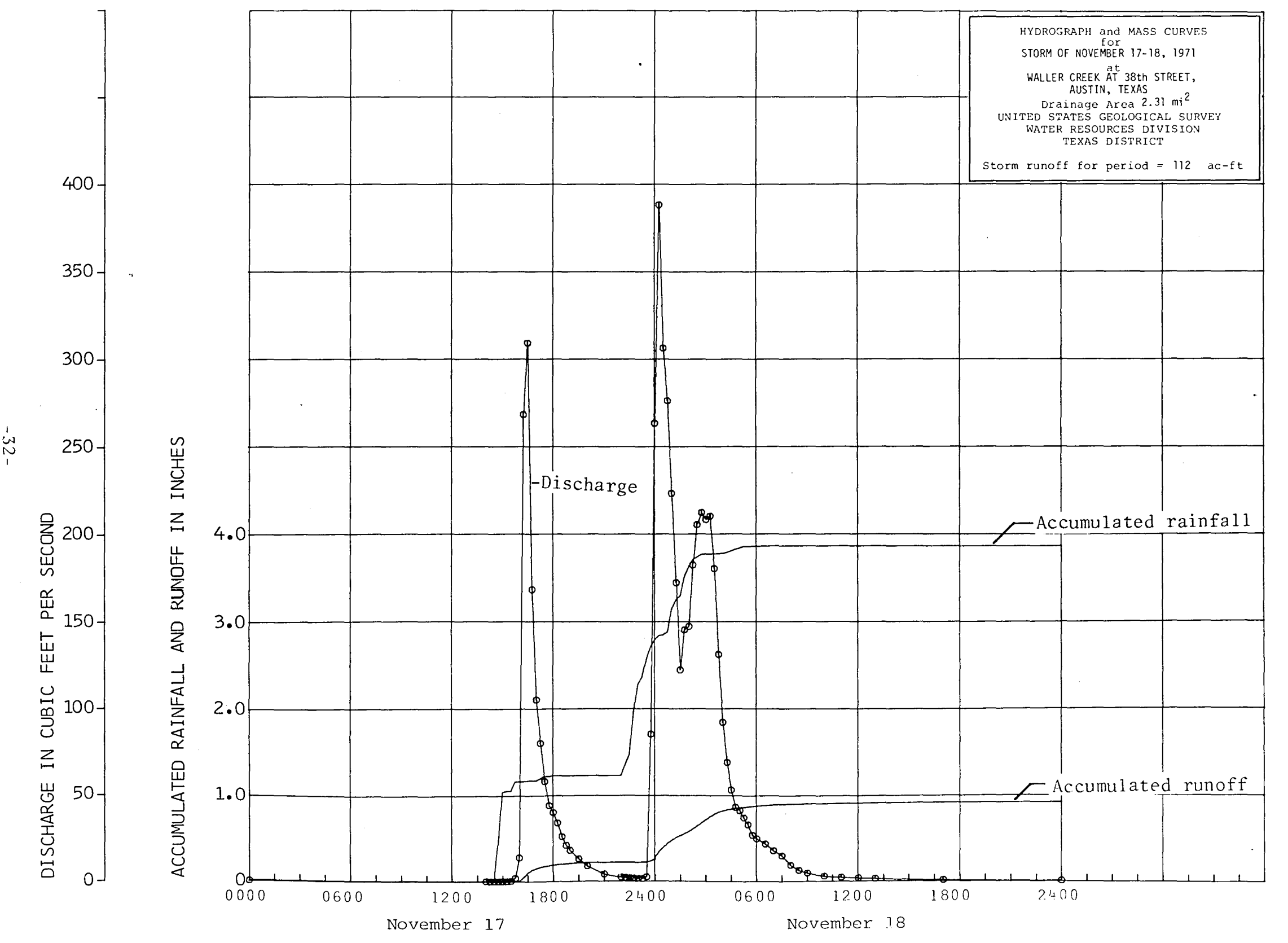




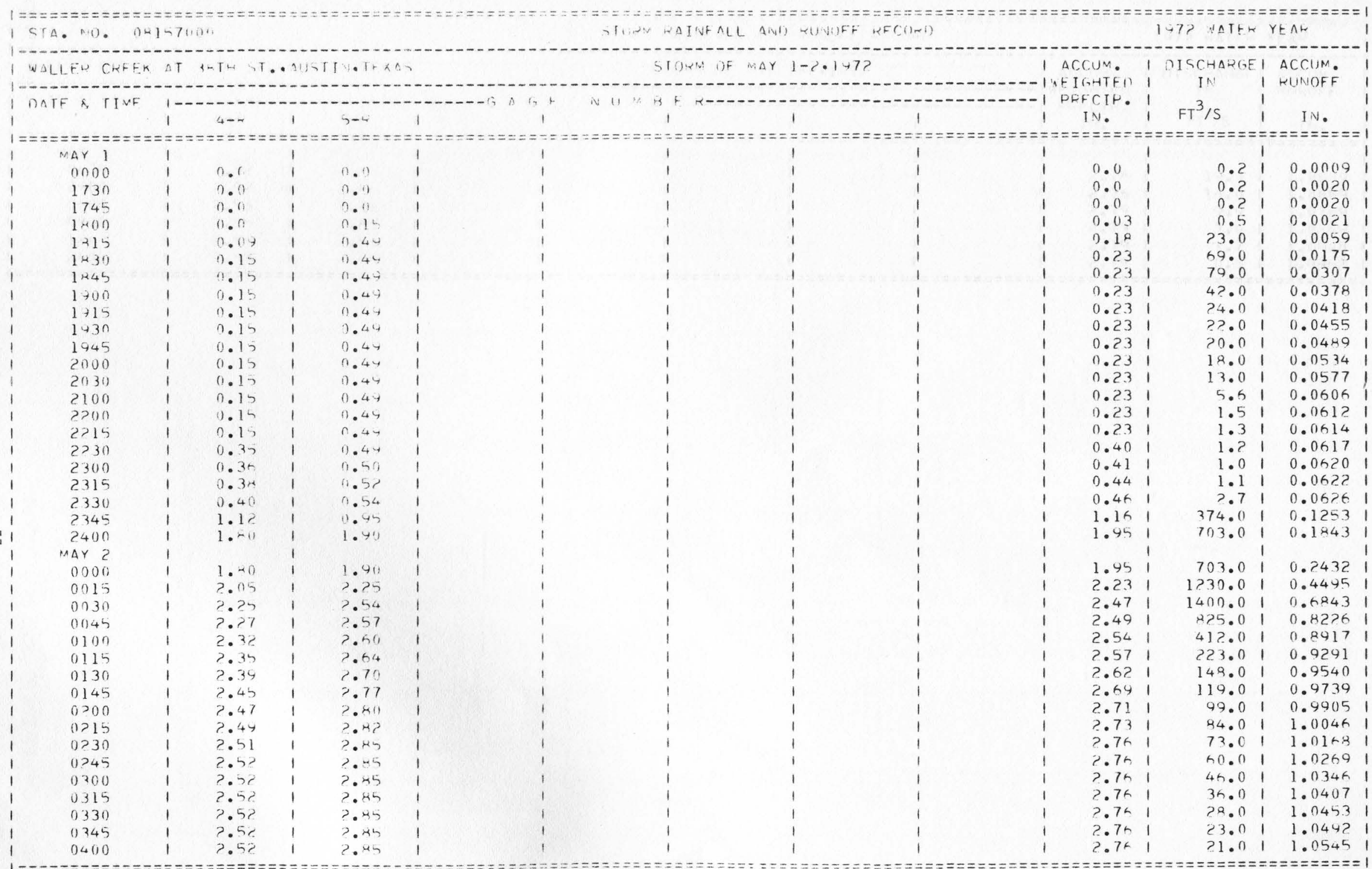





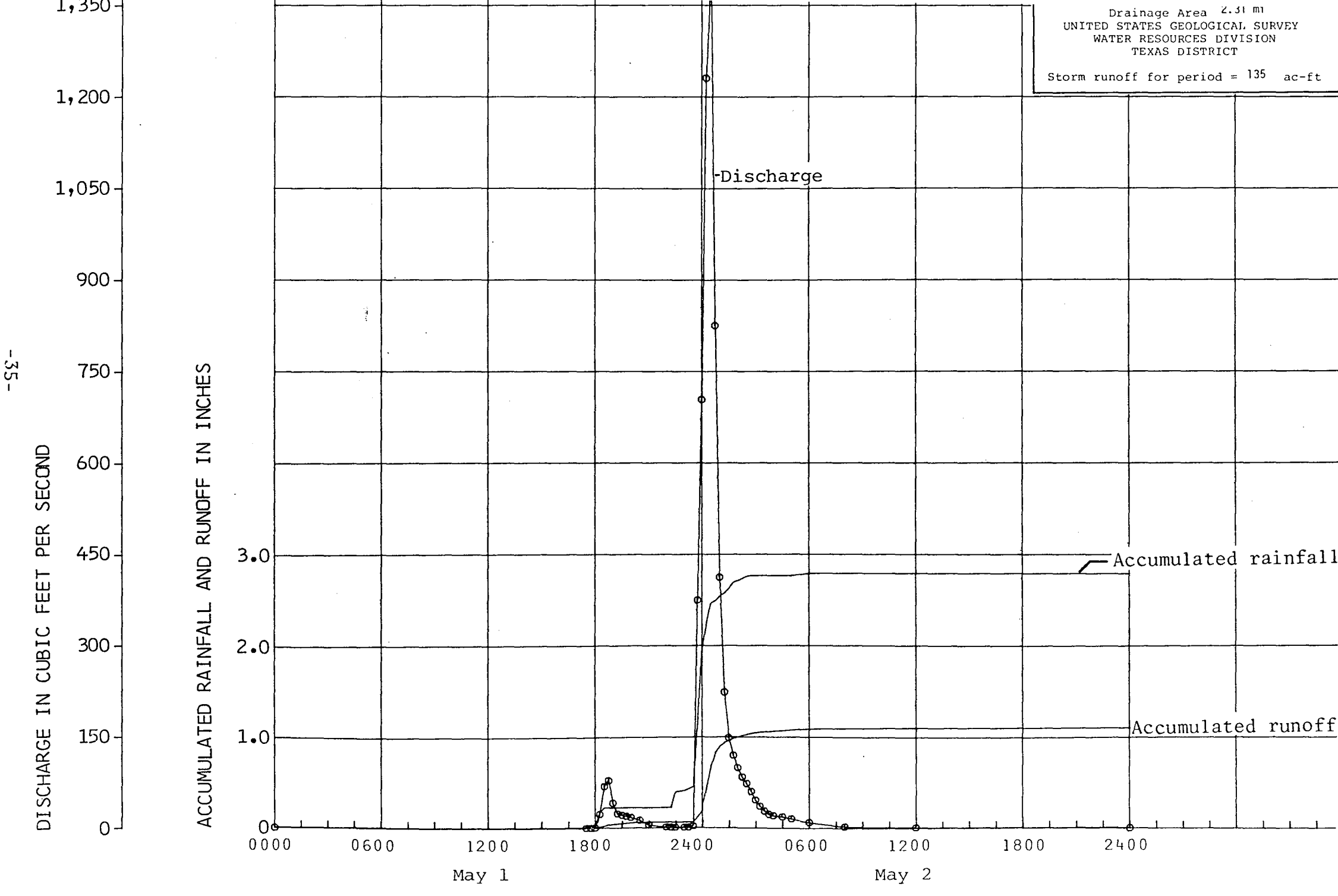




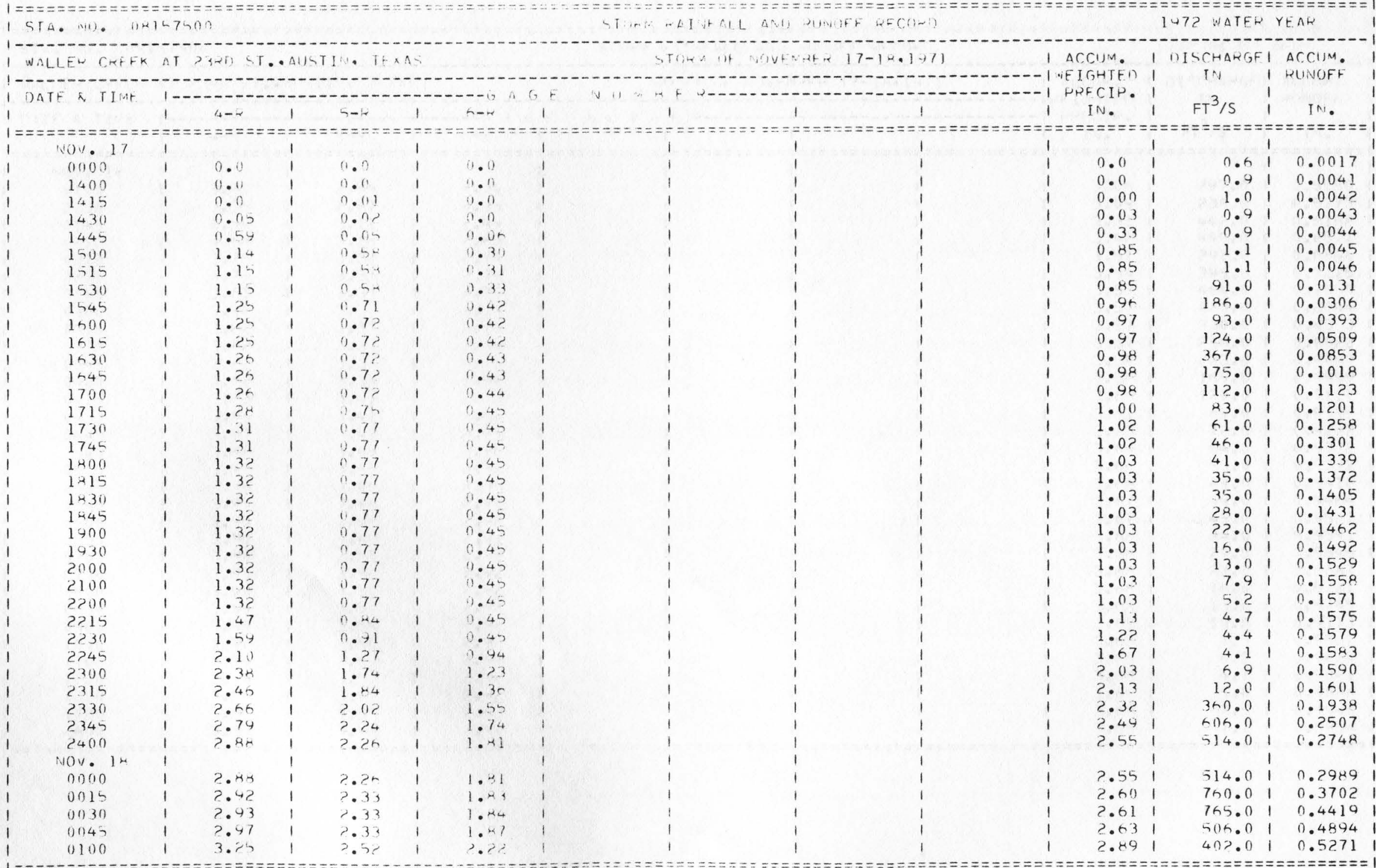




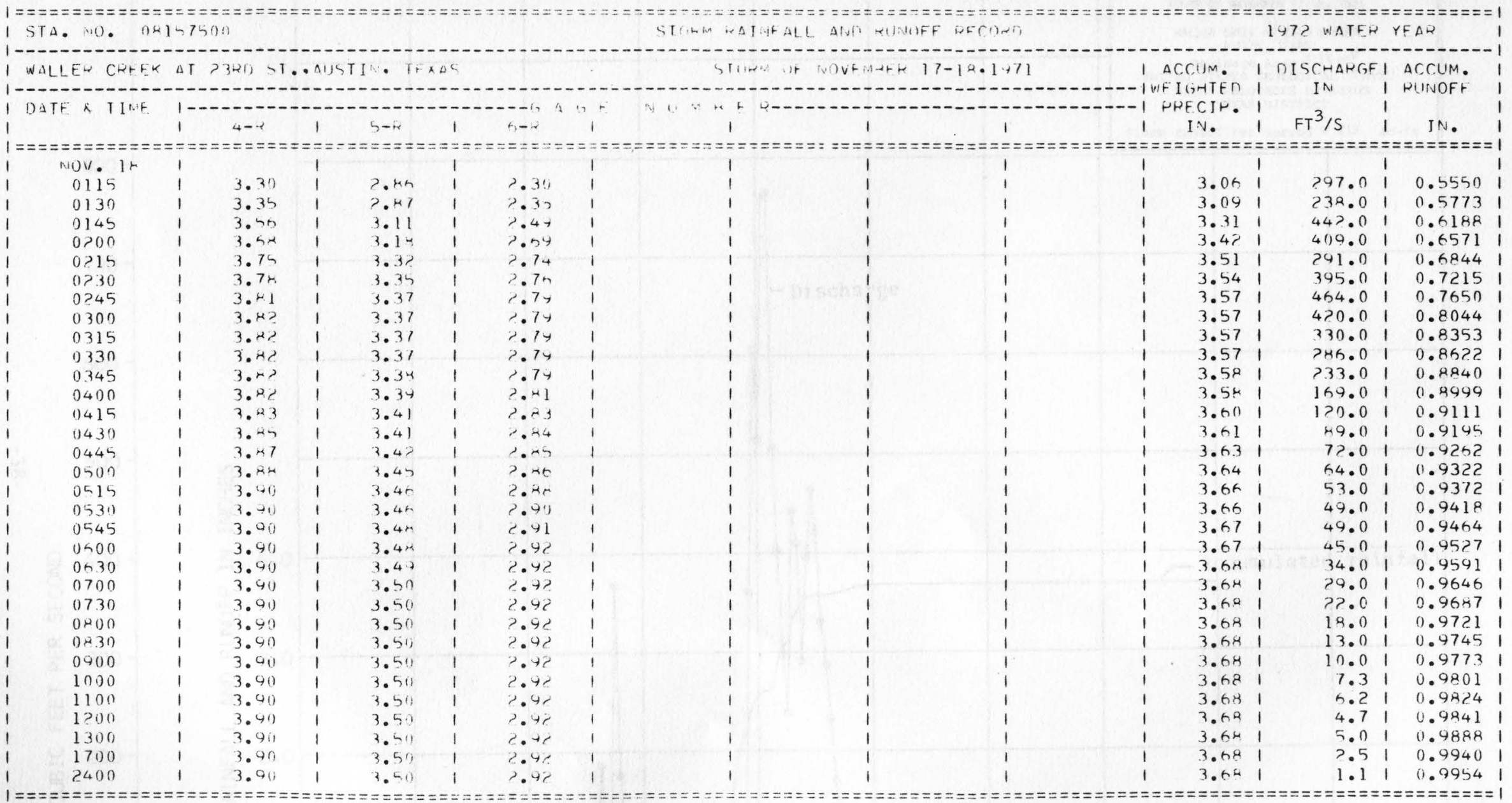




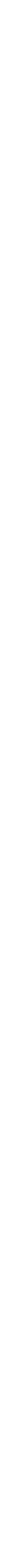




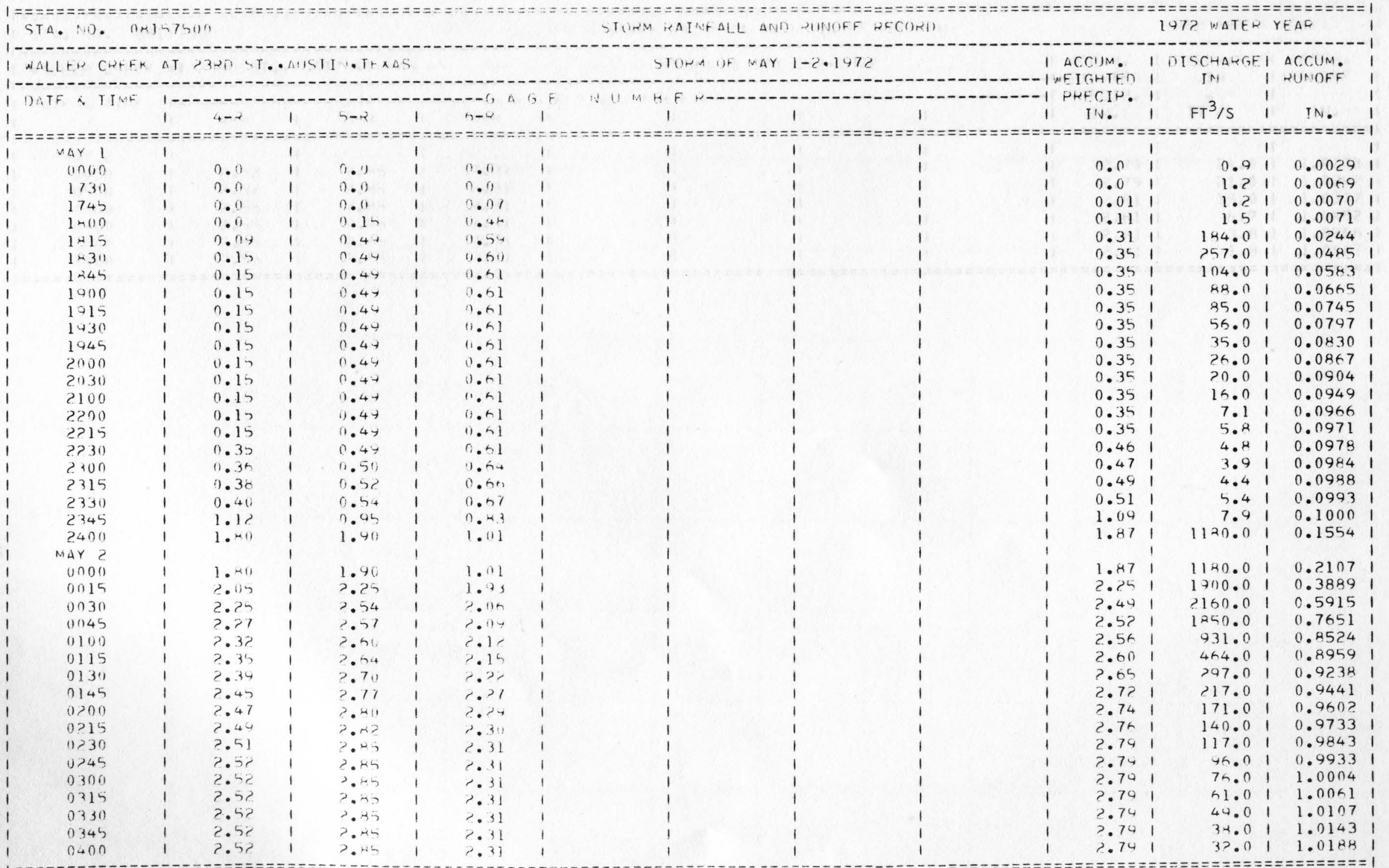




E




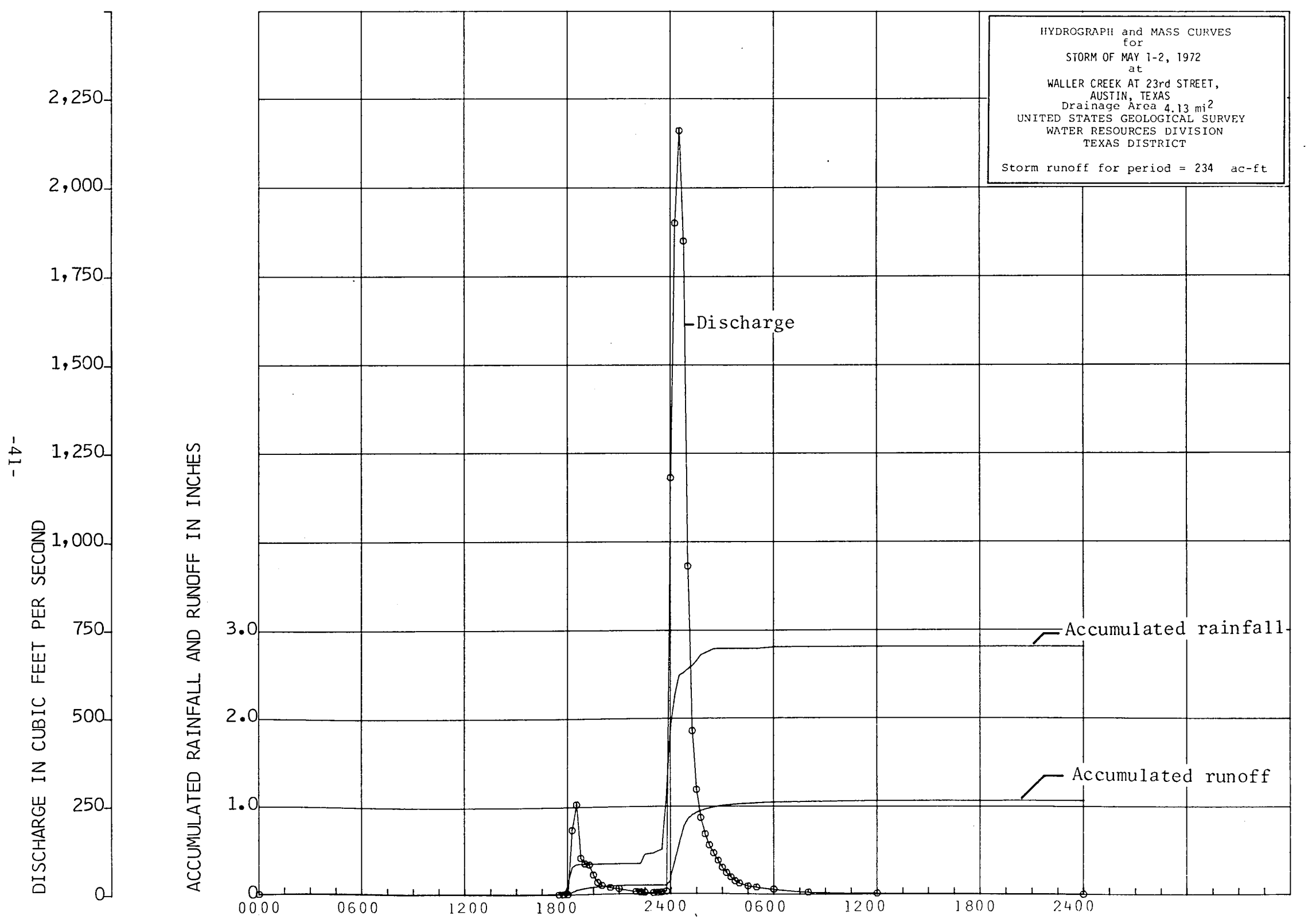




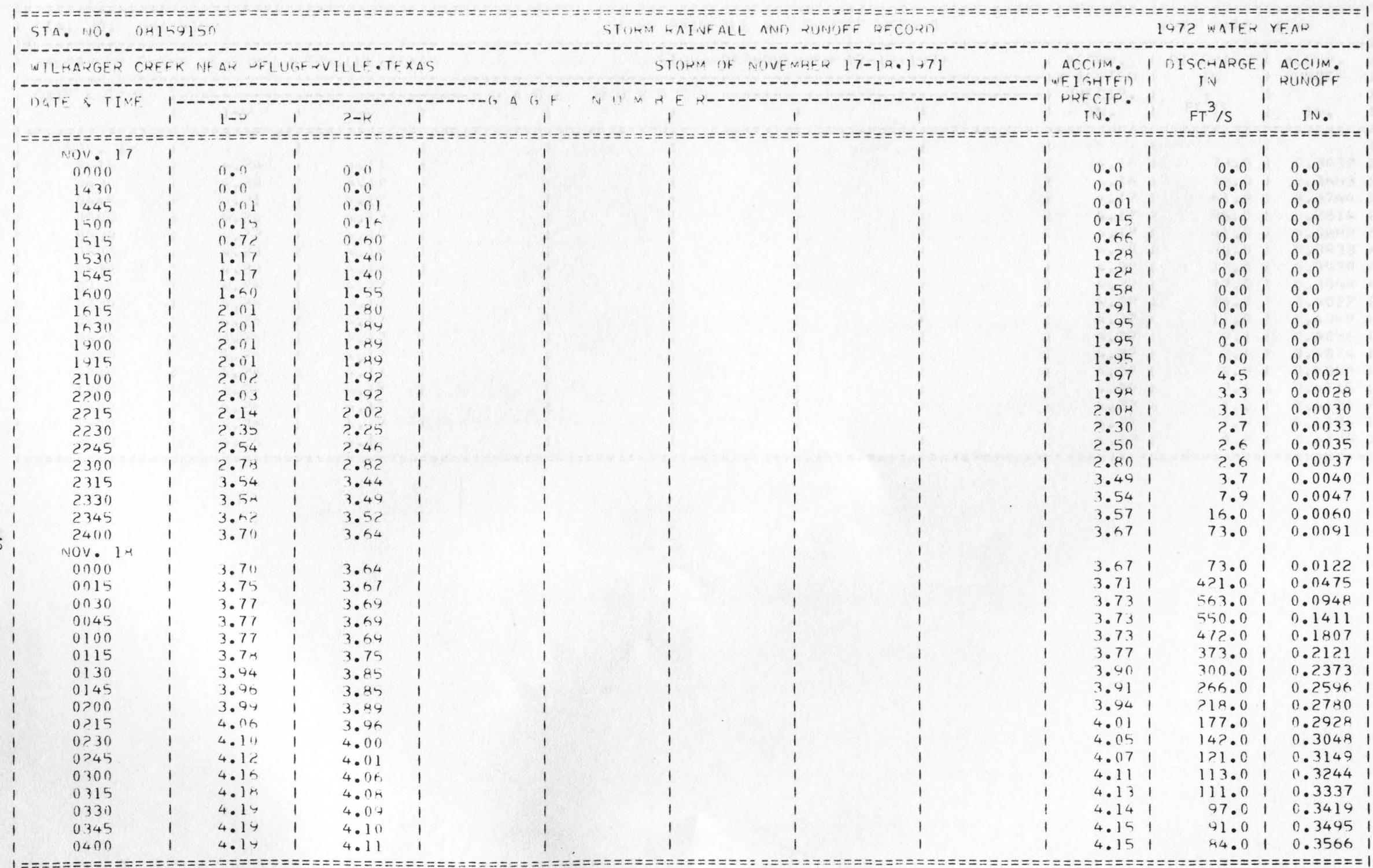




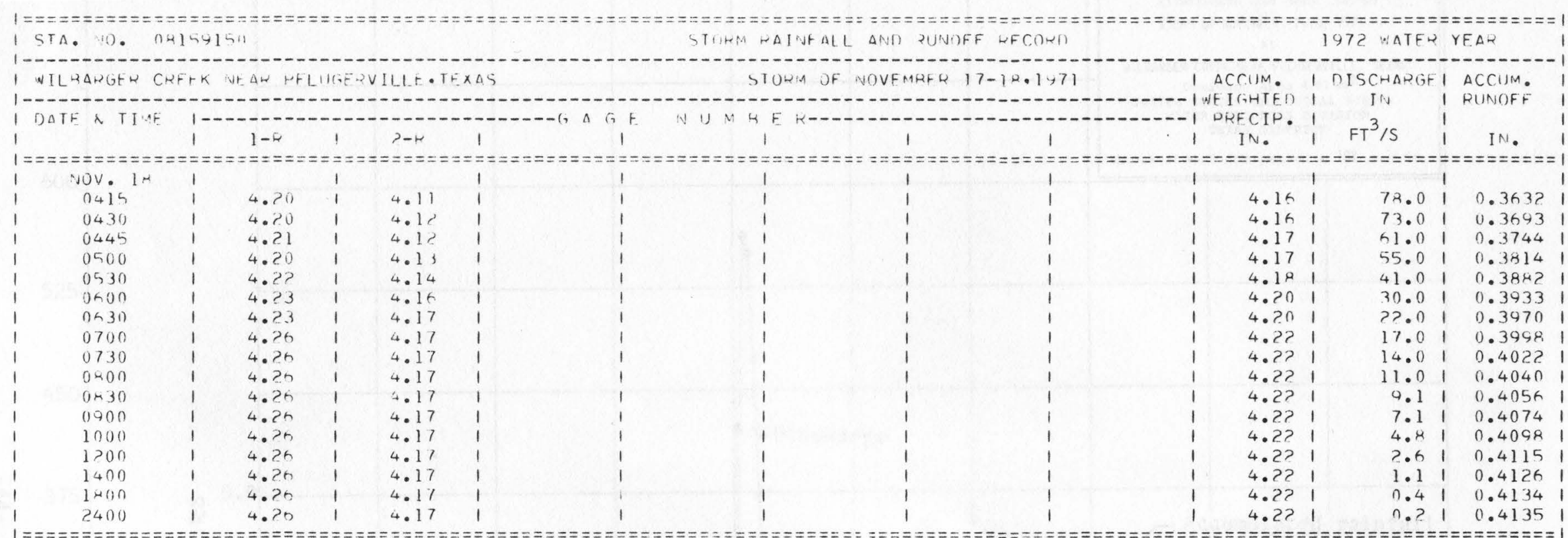




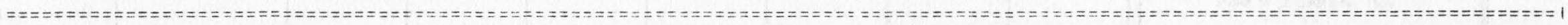

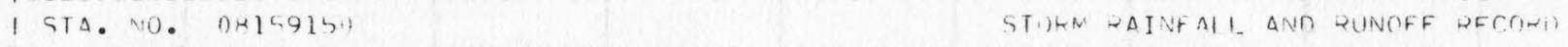
1972 NATEF YEAR

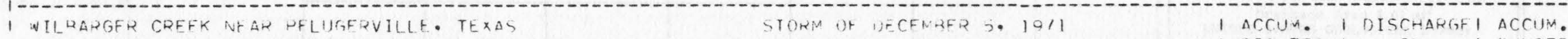

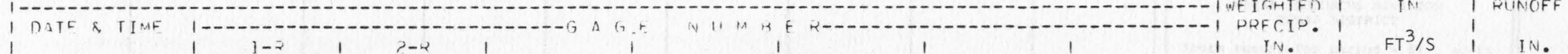

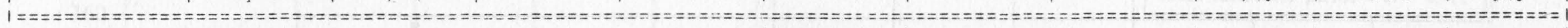

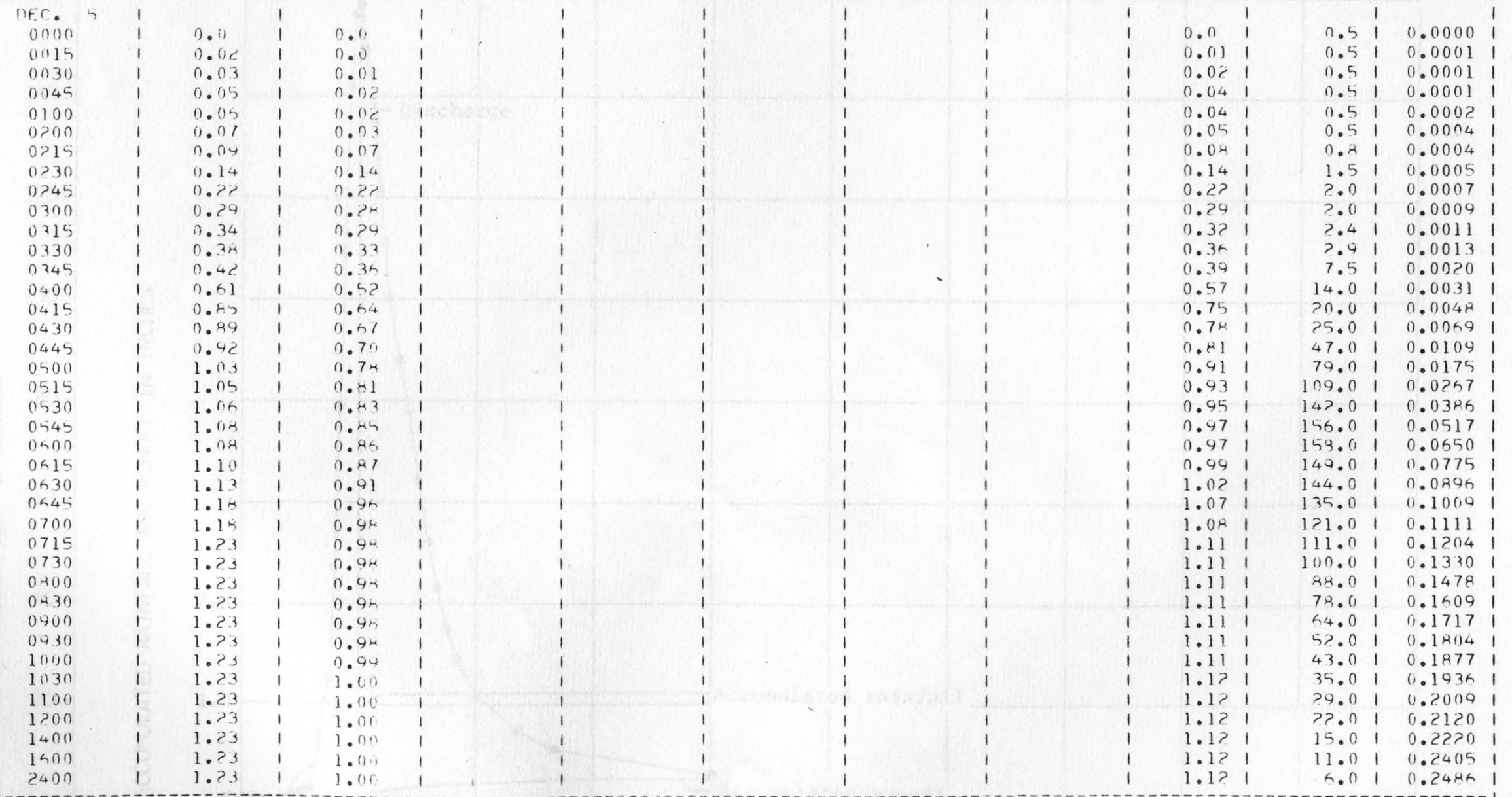




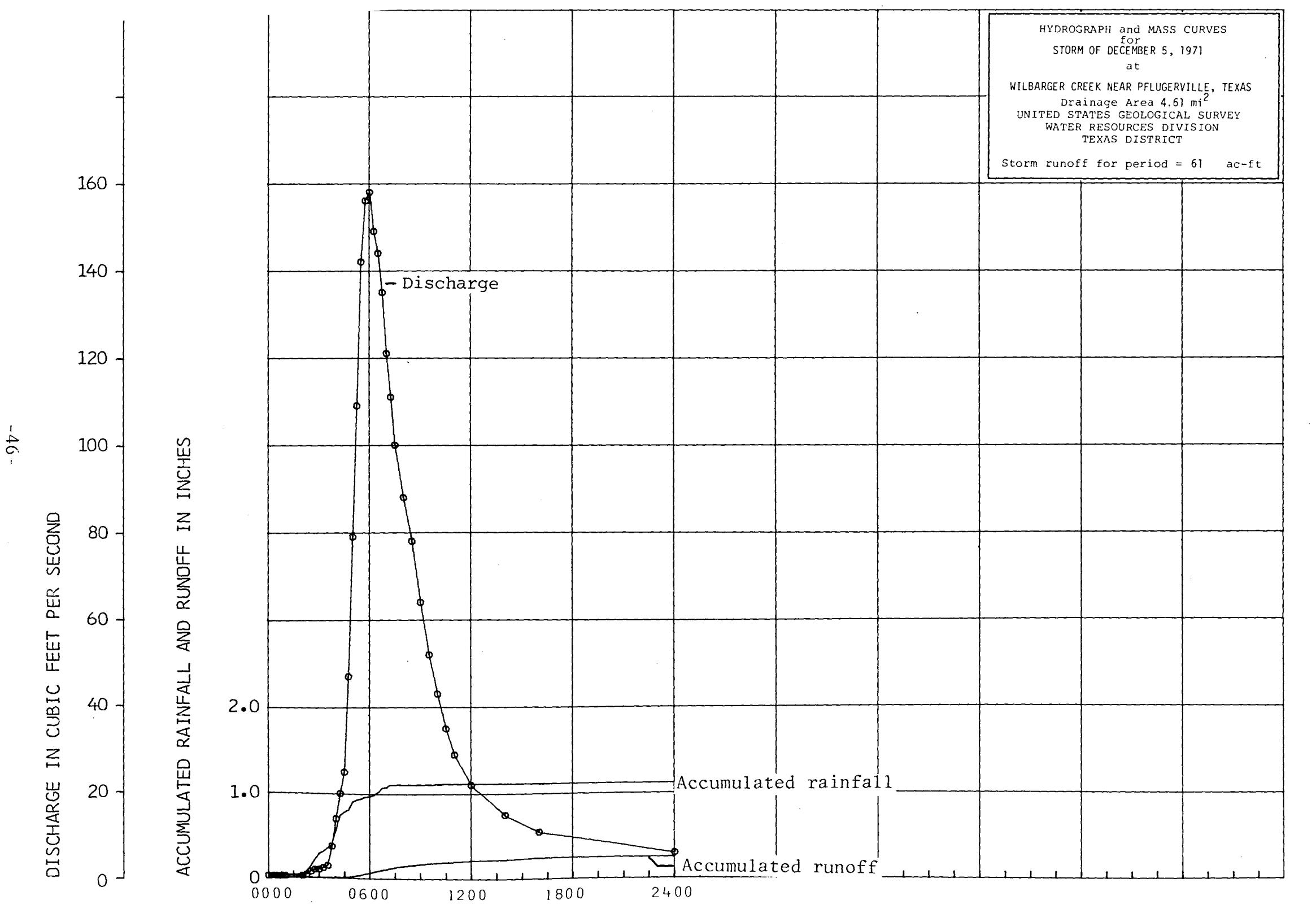




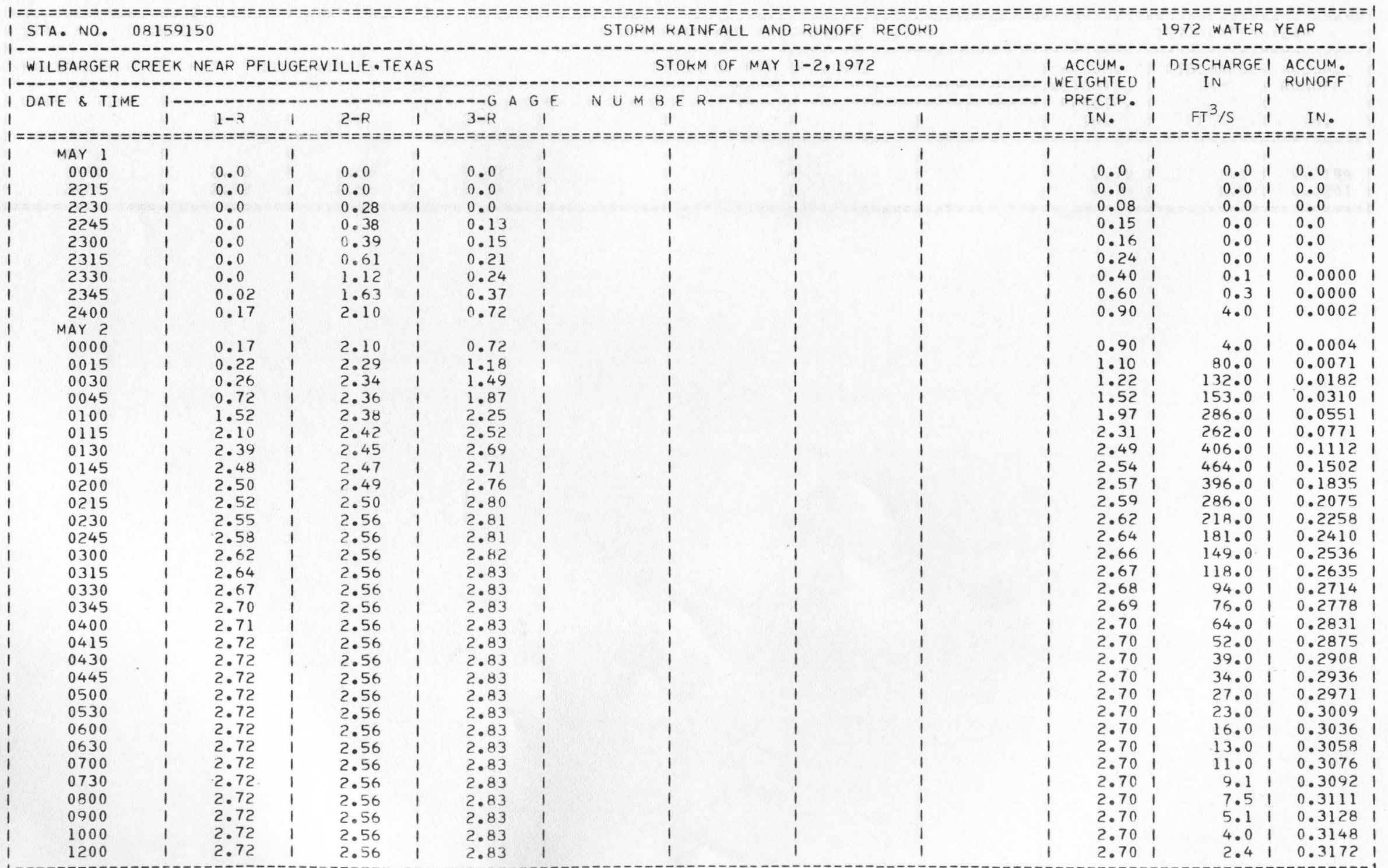




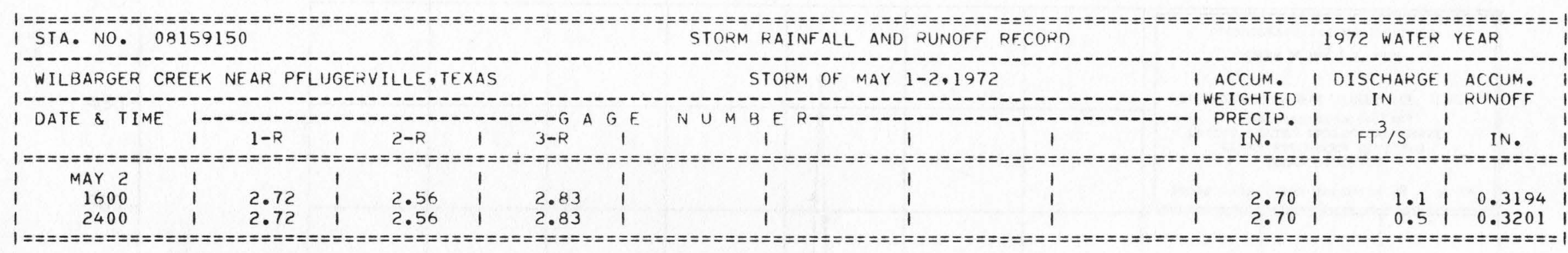




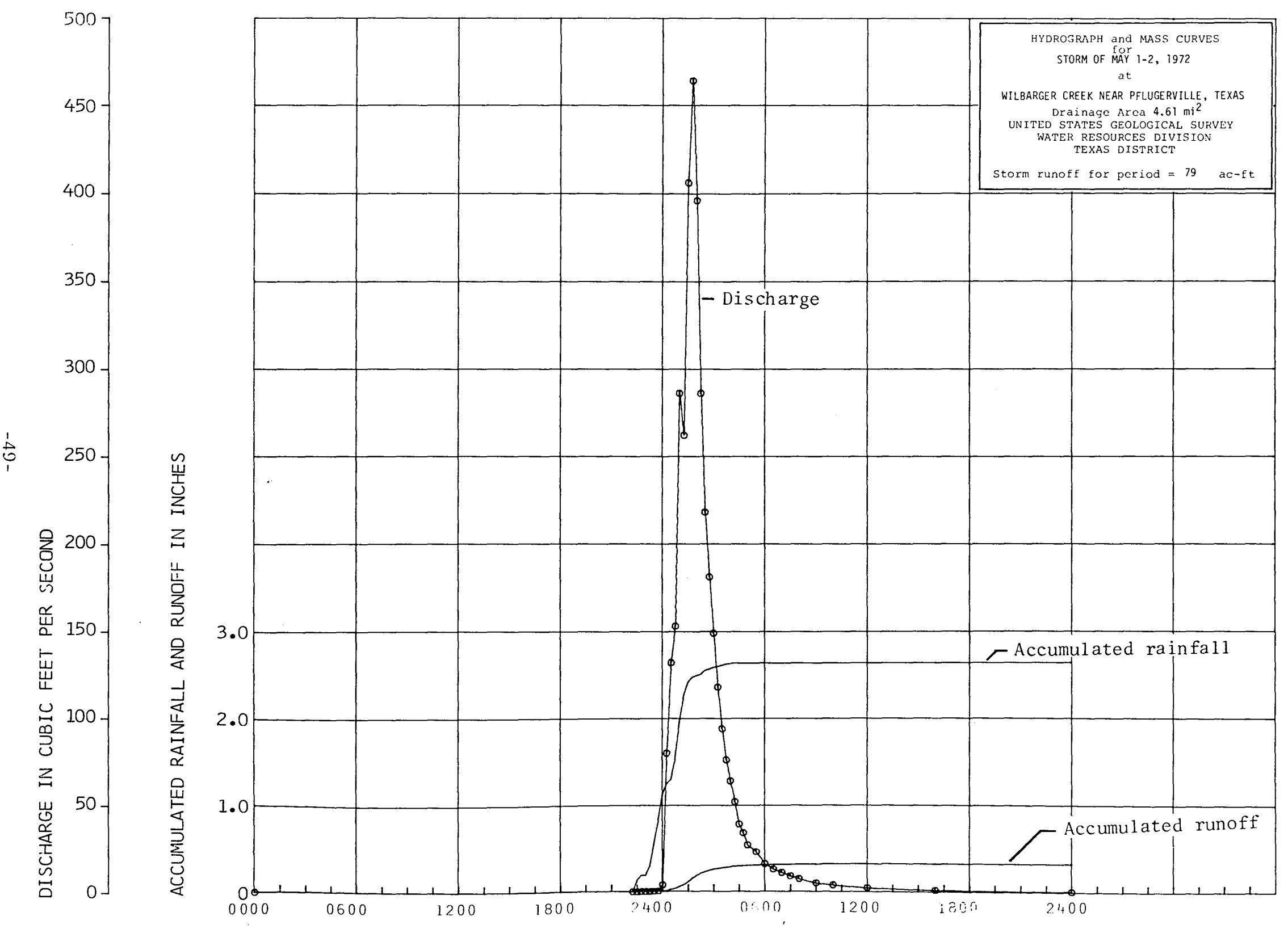

